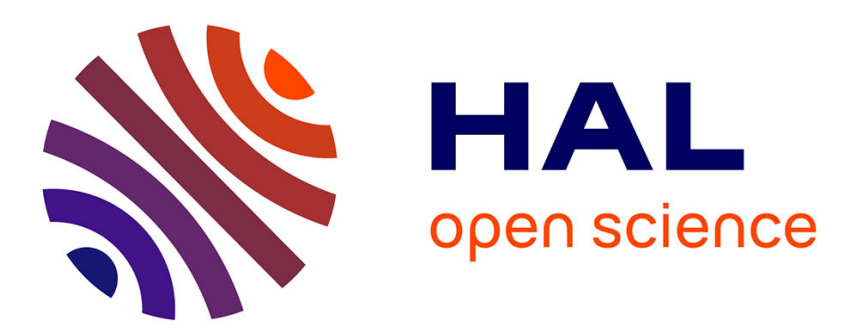

\title{
A new DNS formalism dedicated to turbulent two-phase flows with phase change
}

L. Germes Martinez, Benjamin Duret, J. Reveillon, F.X. Demoulin

\section{To cite this version:}

L. Germes Martinez, Benjamin Duret, J. Reveillon, F.X. Demoulin. A new DNS formalism dedicated to turbulent two-phase flows with phase change. International Journal of Multiphase Flow, 2021, 143, pp.103762. 10.1016/j.ijmultiphaseflow.2021.103762 . hal-03324007

\section{HAL Id: hal-03324007 \\ https://hal.science/hal-03324007}

Submitted on 1 Sep 2021

HAL is a multi-disciplinary open access archive for the deposit and dissemination of scientific research documents, whether they are published or not. The documents may come from teaching and research institutions in France or abroad, or from public or private research centers.
L'archive ouverte pluridisciplinaire HAL, est destinée au dépôt et à la diffusion de documents scientifiques de niveau recherche, publiés ou non, émanant des établissements d'enseignement et de recherche français ou étrangers, des laboratoires publics ou privés. 


\title{
A new DNS formalism dedicated to turbulent two-phase flows with phase change
}

\author{
L. Germes Martinez, B. Duret*, J. Reveillon, F.X. Demoulin \\ CORIA-UMR 6614 - Normandie Université, CNRS-Université et INSA de Rouen, Campus \\ Universitaire du Madrillet, 76800 Saint Etienne du Rouvray, France.
}

\begin{abstract}
Phase change in multiphase flows occurs in many natural and industrial applications, e. g., rain formation, internal combustion engines, heat exchangers, multiphase reactors, etc. In dense two-phase flows, quantitative experimental results are scarce due to the complexity of the configuration and experimental techniques limitations. Hence, the interest in the direct numerical simulation of such flows has grown recently to better understand and access quantitative data in this kind of flows.

When simulating phase change in multiphase flows, advanced numerical method are needed to consider the jump conditions at the interface, and to ensure mass, energy, momentum and species conservation. In the literature, this problem is mainly investigated with an incompressible formalism. However, this assumption is not longer suitable for simulation of multiphase flows with phase change in enclosed environment or in atomized/aerated flows.

The purpose of this work is to present a numerical formalism dedicated to turbulent two phase flows, including acoustics and compressible effects with a proper treatment of the jump conditions at the interface due to phase change. To achieve this task, first, the incompressible level-set method for vaporizing two-phase flows proposed by Tanguy et al. (2007) is revisited and adapted to a mass conservative interface representation: The Coupled Level-set/Volume of Fluid method. In this context, evaporating static cylinder with a constant
\end{abstract}

\footnotetext{
${ }^{*}$ Corresponding author : duret@coria.fr
} 
vaporization rate and a droplet vaporization ( $D^{2}$ law) have been performed as validation cases. Both cases illustrate the method accuracy and robustness in presence of velocity discontinuities at the interface due to the presence of the Stefan flow. The $D^{2}$ law configuration is used as validation of the implementation of the heat and mass transfer transport equations with their jump conditions and the coupling of the evaporation rate with the flow dynamic.

Then, the numerical method is extended to compressible flows using the framework dedicated to the pressure based method proposed by Duret et al. (2018). The main advantage of this framework is the ability to consider acoustics effects, variable density and multiple gas inclusions with its own thermodynamic pressure. A modified Volume of Fluid transport equation is presented, including phase change and compressiblity effects. Navier-Stokes and heat and mass transfer transport equation are solved using compressible assumptions. A validation case of a static evaporating cylinder in an enclosed environment is studied to illustrate and quantify the mass conservation properties of the method and the mass transfer between the two phases. Finally, a 3D two-phase Homogeneous Isotropic Turbulence (HIT) configuration is presented to demonstrate the potential of this method in presence of breakup, gas encapsulation, coalescence and evaporation processes.

Keywords: Compressible, DNS, interface, Two-phase flows, phase change, CLSVOF

\section{Introduction}

Phase change in a multiphase flows is a crucial part of many environmental and industrial processes : from the formation of a raindrop to the fuel vaporization in a internal combustion engine. In addition, multiple types of heat

exchanger rely in the latent energy released or absorbed during the phase change (e.g. cooling towers, spray coolers, evaporators in cooling systems, etc.). Therefore, it highlights the importance of understanding its fundamental behavior for the design and optimization of these types of systems. 
In general, experiments on the heating, evaporation and combustion of droplets are mainly focused on stationary droplets supported by fibers (Mikami et al. (2005), Strizhak et al. (2018), Chauveau et al. (2011)) or droplets in tandem (Kristyadi et al. (2010), Maqua et al. (2008)). For the first case, one of the principal difficulties is the estimation of the fiber role on the temperature distribution of the droplet. This problem has been analyzed by Chauveau et al. (2019), concluding that most fiber techniques used in the literature, which uses relatively large fibers, enhances the droplet evaporation rate due to the increased conduction heat transfer through the fiber. The effects of a turbulent flow on the evaporation rate of a single suspended droplet have been studied by Birouk and Gökalp (2006), Verwey and Birouk (2018), Verwey and Birouk (2017). In the case of droplets in tandem, the main difficulties are associated to the tracking of a falling droplet as it moves away of the camera focus.

Even though great advancement regarding heat and mass transfer measurement techniques have been made in the literature, e. g., Interferometric Particle Imaging (Bilsky et al., 2011), Laser-Induced Fluorescence (Akhmetbekov et al. 2010) and Phase Rainbow Refractometry (Wu et al., 2016), it is still very challenging to quantify the vaporization rate in a dense spray where many liquid structures interact between each other. In that aim, a number of numerical studies has been developed in the past years to go further in the description of the phase change process in dense spray or turbulent liquid jets.

When simulating multiphase flows using a one-fluid formalism, two main approaches to describe the evolution of the interface are commonly used: Interfacetracking and Interface-capturing methods. The last formalism is the most used in the two-phase community (Mirjalili et al. 2017). The Volume of fluid method (VoF) (Hirt and Nichols, 1981) and the Level-set method (LS) (Sussman et al., 1994 belong to this category. Simulation of vaporizing two-phase flows are commonly under one of the following two assumptions: 1) the vaporization rate is computed with the local gradients of the vapor mass fraction (evaporation). Here, the phase change occurs below the boiling temperature (Palmore Jr and Desjardins (2019), Scapin et al. (2020) Wang and Yang (2019), Malan et al. 
(2020), Schlottke and Weigand (2008); 2) the vaporization rate is computed with the heat flow passing through the interface after the saturation temperature has been reached (boiling) (Guion et al. (2018), (Khalloufi et al., 2020), (Urbano et al., 2019), (Popescu et al., 2019), (Tomar et al., 2005) ).

When simulating these types of flows, one of the most challenging problems is the numerical treatment of the different jump conditions at the interface. In particular, the velocity jump condition should satisfy the mass conservation. For example, in the case of a large density ratio and large evaporation rate, the discontinuity in the velocity field close to the interface creates numerical instabilities without proper treatment of the convective terms in the Navier-Stokes solver and in scalars transport equations. In the literature, several numerical techniques have been proposed to solve this issue. Tanguy et al. (2007) proposed a numerical method based on the Ghost Fluid Method (GFM) for the extension of the liquid and gas velocity field. By modifying the method of Nguyen et al. (2001), Tanguy et al. (2007) were able to obtain a continuous and divergence-free liquid velocity field for the advection of the level-set function. Also, Gibou et al. (2007) proposed a similar GFM for treating the velocity jump condition for the simulation of film boiling in 2D. On the other hand, Schlottke and Weigand (2008) proposed a method for the simulation of evaporating droplets using a VoF method. In this work, the liquid and gas velocity fields are computed considering the volume source induced by the evaporation rate to account for the Stefan flow. An iterative algorithm is implemented to compute and distribute the divergence error of the continuity equation to the neighboring cells, this error is due to the difference between the volume and mass averaged velocities at the interface. Later, Reutzsch et al. (2020) improved the method of Schlottke and Weigand (2008) by adding a correction term for the volume source term in the continuity equation which takes into account the variation of the volume and mass averaged velocities. Consequently, the iterative algorithm or the distribution of the error is not longer needed. In the framework of the Vof method, Malan et al. (2020) proposed a method for creating a divergence-free liquid velocity field for the transport of the liquid volume fraction for boiling 
flows. The velocity jump at the interface is subtracted from the one-fluid velocity field. A similar idea has been proposed by Scapin et al. (2020). Palmore Jr and Desjardins (2019) has proposed an alternative way to obtain a divergence-free liquid velocity field: the liquid velocity is extrapolated in the gas phase using the Aslam's extension method (Aslam (2004) $)$ and then the new velocity field is projected onto its divergence-free part using an Helmholtz decomposition.

Until now, the aforementioned studies used an incompressible numerical formalism for the resolution of the Navier-Stokes equations. This assumption is no longer suitable for simulation of multiphase flows with phase change in enclosed environment or with encapsulated gas structures such as atomization configurations. In most of these studies, an outflow boundary condition is used to evacuate the divergence created at every iteration in the gas. As a consequence, the gas density and the thermodynamic pressure remains constant in this kind of approaches. To take into account the increase of gas density and pressure due to vaporization and/or thermal dilatation, a weakly compressible approach should be used. In a previous work of Duret et al. (2018), a pressure based method was developed for the simulation of vaporizing compressible two-phase flows. This formalism was able to describe atomization processes (high Weber and Reynolds numbers), allowing an accurate representation of encapsulated gas structures with their own thermodynamic pressure. However, coupling between the energy and the vaporization rate was not yet implemented. In the literature, studies able to describe this type of problems are very scarce. Wang et al. (2019) proposed a method for modeling the evaporation of two-phase flows while solving the Navier-Stokes equations under a low Mach number limit. More recently, Dodd et al. (2021) study the evaporation of a isolated droplet in a homogeneous isotropic turbulence configuration. They used a low Mach number formulation to solve the Navier-Stokes equations in the gas phase while maintaining the incompressible assumption in the liquid phase. Scapin et al. (2021) proposed a low Mach number formulation for studying the evaporation of droplets in a homogeneous shear turbulence. Nevertheless, they only considered one unique gas structure with a constant thermodynamic pressure. 
This work present numerical strategies for simulating turbulent two-phase flows which take into account the surface tension, acoustic/compressible effects, heating, phase change and large density ratio. We propose to achieve this task by extending the projection method proposed by Tanguy et al. (2007) into a compressible formalism using the weakly-compressible framework proposed by Duret et al. (2018). Note that this formalism is not based on a Low Mach number approximation, but on an All Mach formalism, similar to the one presented by Kwatra et al. (2009), Huber et al. (2015) and Duret et al. (2018). Moreover, this formalism is combined with an accurate and conservative interface capturing method (CLSVOF method), allowing to simulate atomization processes (high Weber and Reynolds numbers). Our approach uses directly the total pressure that depend of the local thermodynamic pressure affected by the vaporization process and the hydrodynamic pressure.

First, the governing equations for a compressible two-phase flow, the interface jump conditions and the numerical strategies are described. Next, validations cases for the incompressible formalism are presented for cylinder (mass conservation) and droplet configuration ( $D^{2}$ law), showing the accuracy and robustness of the method. Then, a simple validation case of an evaporating cylinder in an enclosed environment is presented for the compressible formalism. Finally, a 3D Homogeneous Isotropic Turbulence (HIT) configuration is studied to illustrate the ability of the method to handle compressible turbulent two-phase flows with phase change.

\section{Governing Equations}

In this work, a system of two immiscible fluids is studied; composed by a mono-component liquid and an ideal mixture of gases (e.g. air and vaporized liquid). Thermodynamic equilibrium is assumed at the interface; where mass, energy and momentum are exchanged between both phases. At first, governing equations for the compressible formalism are presented. Then, the additional assumptions leading to incompressible Navier-Stokes equations are exposed. Fi- 
nally, a discussion about the jump conditions for the discontinuous variables and the boundary condition used for the species conservation equation at the interface is presented.

\subsection{Compressible flow}

The Navier-Stokes and pressure equations for a fully compressible flow in one fluid formalism, can be written as:

$$
\left\{\begin{array}{l}
\frac{\partial \rho}{\partial t}+\nabla \cdot(\rho \boldsymbol{u})=0 \\
\frac{\partial \rho \boldsymbol{u}}{\partial t}+\boldsymbol{\nabla} \cdot(\rho \boldsymbol{u} \otimes \boldsymbol{u})=\boldsymbol{\nabla} \cdot \overline{\bar{\Omega}}+\rho \boldsymbol{f}_{\boldsymbol{v o l}} \\
\frac{\partial P}{\partial t}+\boldsymbol{u} \cdot \boldsymbol{\nabla} P=-\rho c^{2} \boldsymbol{\nabla} \cdot \boldsymbol{u}+\frac{\alpha_{T} c^{2}}{c_{p}}(\boldsymbol{\nabla} \cdot(\lambda \boldsymbol{\nabla} T)+\overline{\bar{\tau}}: \boldsymbol{\nabla} \boldsymbol{u}+\dot{Q})
\end{array}\right.
$$

where $\rho, \boldsymbol{u}$ and $P$ are the density, velocity and pressure, respectively. $\boldsymbol{f}_{\boldsymbol{v} o \boldsymbol{l}}$

is the volume forces, $\overline{\bar{\Omega}}=-P \overline{\bar{I}}+\overline{\bar{\tau}}$ is the total stress tensor and $\overline{\bar{I}}$ the identity matrix. The viscous stress tensor is defined as:

$$
\overline{\bar{\tau}}=2 \mu \overline{\bar{\epsilon}}-\frac{2}{3} \mu(\boldsymbol{\nabla} \cdot \boldsymbol{u}) \overline{\bar{I}}
$$

where $\overline{\bar{\epsilon}}=\frac{1}{2}\left(\boldsymbol{\nabla} \boldsymbol{u}+\boldsymbol{\nabla} \boldsymbol{u}^{T}\right) . \quad c$ is the sound speed, $\mu$ the dynamic viscosity, $c_{p}$ is the heat capacity, $\alpha_{T}$ is the coefficient of thermal expansion, $T$ is the temperature, $\lambda$ is the thermal conductivity and $\dot{Q}$ is an energy source term. This set of equation does not include directly phase change terms, but they will be considered in the jump conditions at the interface, described in the following sections.

Here, both fluids are considered to be compressible. Which implies that the system of equations 1 must be closed by two equations of state. For the liquid, a modified version of the Tait's equation is chosen:

$$
\rho_{l}=\rho_{0}\left(\frac{P-P_{0}}{B}+1\right)^{\frac{1}{\gamma_{l}}}
$$

where $\rho_{0}$ is a density at a reference state, $P_{0}$ is a reference pressure, $B$ is a constant and function of the isothermal compressibility of the fluid and $\gamma_{l}$ is a pressure-independent parameter also inherent to the fluid. 
Concerning the gas phase, for isentropic case, such as the configuration presented in Section 5.1, a Laplace's law is chosen:

$$
\rho_{g}=\left(\frac{P}{C_{\gamma}}\right)^{\frac{1}{\gamma_{g}}}
$$

where $\gamma_{g}$ is the heat capacity ratio and $C_{\gamma}$ is a constant dependent of the initial condition of the ideal gas.

In compressible configurations with heat and mass transfer, such as the HIT configuration in Section 5.2, a perfect gas Equation of state is used :

$$
\rho_{g}=\frac{P}{r T}
$$

where $r$ is the specific ideal gas constant and $P$ the total pressure.

The energy conservation equation can be expressed in term of the temperature:

$$
\rho c_{p}\left(\frac{\partial T}{\partial t}+\boldsymbol{u} \cdot \nabla T\right)=\boldsymbol{\nabla} \cdot(\lambda \nabla T)+\alpha_{T} T\left(\frac{\partial P}{\partial t}+\boldsymbol{u} \cdot \nabla P\right)+\overline{\bar{\tau}}: \nabla \boldsymbol{u}+\dot{Q}
$$

A similar set of equations for compressible two-phase flows is presented in Caltagirone et al. (2011).

Since the liquid phase is considered as mono-component, the species conservation equation is only described for the gas phase:

$$
\frac{\partial \rho_{g} Y_{v}}{\partial t}+\nabla \cdot\left(\rho_{g} \boldsymbol{u} Y_{v}\right)=\boldsymbol{\nabla} \cdot\left(\rho_{g} D_{m} \boldsymbol{\nabla} Y_{v}\right)
$$

where $Y_{v}$ is the vapor mass fraction and $D_{m}$ is the mass diffusivity.

\subsection{Incompressible flow}

In the incompressible part of this work, the fluid velocity field is computed using equations 1, with a divergence-free condition in both phases:

$$
\left\{\begin{array}{l}
\boldsymbol{\nabla} \cdot \boldsymbol{u}=0 \\
\frac{\partial \boldsymbol{u}}{\partial t}+(\boldsymbol{u} \cdot \boldsymbol{\nabla}) \boldsymbol{u}=-\frac{\boldsymbol{\nabla} P}{\rho}+\frac{2 \mu \overline{\bar{\epsilon}}}{\rho}+\boldsymbol{f}_{\boldsymbol{v o l}} \\
\rho c_{p}\left(\frac{\partial T}{\partial t}+\boldsymbol{u} \cdot \boldsymbol{\nabla} T\right)=\boldsymbol{\nabla} \cdot(\lambda \boldsymbol{\nabla} T) \\
\frac{\partial Y_{v}}{\partial t}+\boldsymbol{\nabla} \cdot\left(\boldsymbol{u} Y_{v}\right)=\boldsymbol{\nabla} \cdot\left(D_{m} \boldsymbol{\nabla} Y_{v}\right)
\end{array}\right.
$$


Here, the density of each phase is considered constant in time and space. In this case, the pressure is a numerical parameter which allow the system to satisfy the divergence-free condition. Hence, there is no need of an additional equation dedicated to the pressure.

Note that it is possible to consider the gas incompressible in configurations with phase change: it has been done in previous work in the literature (Tanguy et al., 2007, Schlottke and Weigand, 2008, Gibou et al., 2007). Under this assumption, the gas density remains constant. However, the velocity divergence produced by the Stefan flow at the interface must be evacuated of the domain, e. g., with an outflow boundary condition.

\subsection{Interface jump conditions}

Considering that we are working with a one-fluid formalism, appropriate jump conditions must be defined for the discontinuous variables at the interface. Commonly for the inert interface, we have:

$$
\begin{aligned}
{[\rho]_{\Gamma} } & =\rho_{l}-\rho_{g} \\
{[\mu]_{\Gamma} } & =\mu_{l}-\mu_{g}
\end{aligned}
$$

and the surface tension is taken into account by the pressure jump at the interface:

$$
[\boldsymbol{n} \cdot(\overline{\bar{\Omega}} \cdot \boldsymbol{n})]_{\Gamma}=\sigma \kappa
$$

with $\sigma$ as the the surface tension, $\kappa$ is the total curvature and $\boldsymbol{n}$ is the vector normal to the interface.

When heat and mass transfer are considered across the interface, additional jump conditions are required to fully describe our system. The mass conser-

vation condition across the interface can be expressed as ( $($ Calimez, 1998$)$ and Ishii and Hibiki (2010) ) :

$$
\rho_{l}\left(\boldsymbol{u}_{l}^{\Gamma}-\boldsymbol{u}^{\Gamma}\right) \cdot \boldsymbol{n}=\rho_{g}\left(\boldsymbol{u}_{g}^{\Gamma}-\boldsymbol{u}^{\Gamma}\right) \cdot \boldsymbol{n}=\dot{\omega}
$$


where $\dot{\omega}$ represent the mass flow across the interface due to evaporation. $\boldsymbol{u}_{\Gamma}$ is the velocity of the interface; which can be described, from the liquid side, as the sum of the liquid velocity at the interface $\left(\boldsymbol{u}_{l}^{\Gamma}\right)$ and the interface regression speed $\left(s_{\boldsymbol{d}}\right)$ :

$$
\boldsymbol{u}^{\Gamma}=\boldsymbol{u}_{l}^{\Gamma}-\left\|\boldsymbol{s}_{\boldsymbol{d}}\right\| \boldsymbol{n}
$$

$s_{\boldsymbol{d}}$ is colinear to the interface normal and defined as the difference of the normal components of $\boldsymbol{u}^{\Gamma}$ and $\boldsymbol{u}_{l}^{\Gamma}$ :

$$
\boldsymbol{s}_{\boldsymbol{d}}=\left(\left(\boldsymbol{u}^{\Gamma}-\boldsymbol{u}_{l}^{\Gamma}\right) \cdot \boldsymbol{n}\right) \cdot \boldsymbol{n}
$$

from eq. 12, we have:

$$
s_{\boldsymbol{d}}=-\frac{\dot{\omega}}{\rho_{l}} \boldsymbol{n}
$$

$\boldsymbol{u}^{\Gamma}$ can be also be defined from the gas phase as:

$$
\boldsymbol{u}^{\Gamma}=\boldsymbol{u}_{g}^{\Gamma}-\frac{\rho_{l}}{\rho_{g}}\left\|\boldsymbol{s}_{\boldsymbol{d}}\right\| \boldsymbol{n}
$$

The magnitude of difference the between equations 13 and 16 is the velocity jump across the interface:

$$
\Delta u=\frac{\rho_{l}-\rho_{g}}{\rho_{g}}\left\|s_{\boldsymbol{d}}\right\|
$$

Also, the thermal conductivity is discontinuous at the interface: $[\lambda]_{\Gamma}=$ $\lambda_{l}-\lambda_{g}$. Additionally, in the presence of phase change, an extra energy jump condition must be considered to respect the energy conservation equation:

$$
\lambda_{l} \boldsymbol{\nabla} T_{l}^{\Gamma} \cdot \boldsymbol{n}-\lambda_{g} \boldsymbol{\nabla} T_{g}^{\Gamma} \cdot \boldsymbol{n}=-h_{l v} \dot{\omega}
$$

where $h_{l v}$ is the latent heat.

Finally, to respect the species conservation, for a monocomponent liquid, the jump condition at the interface for $Y_{v}$ is:

$$
\left.\rho_{g} D_{m} \boldsymbol{\nabla} Y_{v} \cdot \boldsymbol{n}\right|_{g} ^{\Gamma}=\dot{\omega}\left(1-Y_{v s}\right)
$$


Where $Y_{v s}$ is the vapor mass fraction at the interface in the gaseous phase. To estimate $Y_{v s}$, the thermodynamic equilibrium is assumed at the interface, allowing a direct relation between the energy and $Y_{v}$. The Clausius-Clapeyron relation is used:

$$
X=\exp \left[-\frac{h_{l v} M_{v a p}}{R}\left(\frac{1}{T^{\Gamma}}-\frac{1}{T_{B}}\right)\right]
$$

where $X$ is the vapor mole fraction at the interface, $M_{\text {vap }}$ is the molar mass of the vapor, $R$ is the ideal gas constant, $T^{\Gamma}$ is the temperature of the interface and $T_{B}$ is the boiling temperature. From the mass definition, $Y_{v s}$ can be calculated as:

$$
Y_{v s}=\frac{X M_{v a p}}{X M_{v a p}+(1-X) M_{g}}
$$

where $M_{g}$ is the molar mass of the inert gas. The denominator of the right hand side of eq. 21 represents the mean molar mass of the gas mixture.

The interface jump conditions of the different variables reveal the strong coupling between heat/mass transfer and the velocity jump. Handling this coupling is challenging and require robust and accurate numerical methods dedicated to DNS of two-phase flow with phase change.

\section{Numerical Methods}

This section is devoted to discuss about numerical strategies used to perform direct numerical simulation of multiphase flows with phase change. When dealing with this kind of flows, numerous difficulties appears due to the diversity of physical processes that takes place simultaneously. In addition, special care is required when implementing the jump conditions at the interface described in the previous section.

In the literature, the numerical representation of an inert interface is well known. To include the phase change phenomenon, these strategies are adapted to consider the interface regression velocity induced by the vaporization of the liquid. Usually, when an interface capturing method is used (e. g., Volume of Fluid (VoF) method or a Level-set (LS) method) this adaptation is done explicitly by adding a sink term in the VoF transport equation or implicitly by 
adding the interface regression speed, defined by eq. 15, to the velocity field used for the convective transport of the scalar.

Furthermore, a discontinuity in the velocity field occurs across the interface (eq. 17). Note that most numerical methods dedicated to the convective transport of the scalars in DNS of two-phases flows are originally developed to handle continuous and divergence-free velocity fields. Hence, they cannot used this velocity field directly. Otherwise, unphysical deformation of the interface will occurs due to an oscillatory behavior of the numerical scheme, which will lead to errors in the mass conservation. Several strategies has been proposed to overcome this issue. Most numerical strategies share the same idea: create a divergence-free velocity field, dedicated to the convective transport of the interface capturing methods.

To extend the applicability of our in-house code ARCHER to reactive interfaces in compressible multiphase flows, we investigated various methods found in the literature. First, the method proposed by Malan et al. (2020) and then modified by Scapin et al. (2020). This formalism is developed for incompressible configurations. The idea of this method is to compute a divergence-free liquid-velocity extension $\left(\boldsymbol{u}_{l}\right)$ for advecting of the liquid volume fraction. This extension is computed by subtracting the velocity jump at the interface from the original velocity field:

$$
\boldsymbol{u}_{l}=\boldsymbol{u}-\tilde{\boldsymbol{u}}
$$

An intermediate velocity field $(\tilde{\boldsymbol{u}})$ is computed, which contain a velocity jump at the interface with the same magnitude that the real velocity field $(\boldsymbol{u})$ but with opposite sign. An advantage of this method is its simplicity of implementation in a VoF solver. But with this method, when the Stefan flow is large, spurious velocities are created due to small errors in the construction of $\boldsymbol{u}_{l}$, which deform the interface in a unphysical way in the long term in our implementation. Then, the method proposed by Palmore Jr and Desjardins (2019) was investigated. Similar to the previous method, it is conceived for a incompressible formalism and its purpose is to construct a divergence-free velocity field to advect the 
liquid volume fraction. In this case, Palmore Jr and Desjardins (2019) takes the original velocity field and extend linearly the velocity of the liquid phase into the gas phase using the Aslam's extension method (Aslam, 2004). As mentioned by the authors, the extended velocity field $\left(\boldsymbol{u}_{l}^{*}\right)$ is not divergence-free leading to erroneous variations in the liquid volume. To overcome this problem, $\boldsymbol{u}_{l}^{*}$ is projected onto its divergence-free part using:

$$
\boldsymbol{u}_{l}=\boldsymbol{u}_{l}^{*}+\nabla \cdot W
$$

where $W$ is a potential derived from the Helmholtz-type equation,

$$
a W+\nabla \cdot W=\nabla \cdot \boldsymbol{u}_{l}^{*}
$$

where $a$ is zero for all liquid-containing cells and cells that are within three grid cells of the interface. The application of this method imply an important increase in the computational cost due to the addition of the Aslam's extension method and the extra Poisson solver. Similar to the previous method, the spurious currents in $\boldsymbol{u}_{\boldsymbol{l}}$ deformed the interface when the vaporization rate is large. A probable reason why the results obtained with aforementioned methods are not satisfactory may that the resulting real velocity field, with the velocity jumps at the interface, is not suitable for the numerical methods used in our DNS code (ARCHER) for the convective and diffusive terms of the Navier-Stokes equation.

Finally, the third method that we are going to analyze and use in the following sections is the one proposed by (Tanguy et al., 2007). With this approach, the Ghost Fluid Method is used for the treatment of the jump condition at the interface given by eq. 17. In this case, the convective terms in transport equations use either the liquid or the gas velocity fields, depending of the sign of the Level Set function. Using the Ghost Fluid Method to populate the ghost cells on each side of the interface, we obtain:

$$
\begin{aligned}
& \boldsymbol{u}_{l}^{\text {ghost }}=\boldsymbol{u}_{g}+\boldsymbol{s}_{\boldsymbol{d}}\left(\frac{\rho_{l}-\rho_{g}}{\rho_{g}}\right) \\
& \boldsymbol{u}_{g}^{\text {ghost }}=\boldsymbol{u}_{l}-\boldsymbol{s}_{\boldsymbol{d}}\left(\frac{\rho_{l}-\rho_{g}}{\rho_{g}}\right)
\end{aligned}
$$


This way we can obtain a liquid velocity field $\left(\boldsymbol{u}_{l}\right)$ without the velocity jump at the interface. In the method of (Tanguy et al., 2007), additional steps have been proposed for ensuring the divergence-free condition in $\boldsymbol{u}_{l}$ for a precise evolution of the interface. In our formalism, this projection method is then improved to include acoustics and compressible effects and combined with a mass conservative interface capturing method (CLSVOF method). These improvements are presented and discussed in the following sections.

\subsection{Interface capturing method}

The Coupled Level-set/Volume of Fluid (CLSVOF) method implemented in the ARCHER code (Ménard et al. 2007) is extended to handle multiphase flows with phase change. In this section, the VoF equation for the compressible formalism with phase change is presented. With this approach, density variations due to temperature, pressure gradients and evaporation in enclosed environments can be captured.

To demonstrate the VoF equation, the first step is to define the density as a weighted mean between the density of each phase:

$$
\rho=\alpha_{l} \rho_{l}+\alpha_{g} \rho_{g}
$$

where $\alpha_{l}$ and $\alpha_{g}$ are the local liquid and gas volume fraction, respectively. Then, the new definition of $\rho$ is introduced into the continuity equation (eq. 1a). Using the conventional notation $\frac{D[\cdot]}{D t}=\frac{\partial[\cdot]}{\partial t}+\boldsymbol{u} \cdot \nabla[\cdot]$ for the material derivative and subtracting a sink term to satisfy mass conservation in the presence of phase change, we can find that:

$$
\frac{\partial \alpha_{l}}{\partial t}+\nabla \cdot\left(\alpha_{l} \boldsymbol{u}_{l}\right)=-\frac{\alpha_{l}}{\rho_{l}} \frac{D \rho_{l}}{D t}-\frac{\dot{m}}{\rho_{l}}
$$

where $\dot{m}$ represent the quantity of liquid volume evaporated in each cell containing an interface. $\dot{m}$ is estimated by:

$$
\dot{m}=\rho_{l} \Sigma\left\|\boldsymbol{s}_{\boldsymbol{d}}\right\|
$$


where $\Sigma=\frac{S}{V_{c e l l}}$ is the surface density in a mixed cell, $S$ is the surface, obtained in the PLIC reconstruction. The material derivative of the density can be obtained by writing eq. $1 \mathrm{a}$ on a non-conservative form and using eq. $1 \mathrm{c}$ for the velocity divergence:

$$
\frac{D \rho_{l}}{D t}=\frac{1}{c_{l}^{2}} \frac{D P}{D t}-\frac{\alpha_{T l}}{c_{p l}} \nabla \cdot(\lambda \nabla T)
$$

The pressure and temperature term of eq. 28 are solved after the computation of the Poisson equation for the pressure and during the evolution of the temperature, respectively. Both of them will be detailed in the next sections.

Since the liquid evaporation is taken into account explicitly by adding a sink term in the RHS of eq. 26, local undershoots of the liquid volume fraction $\left(\alpha_{l}<0\right)$ can occur. To respect mass conservation and ensure an accurate interface regression due to the evaporation, the extra fraction of evaporated liquid is distributed to the neighboring grid cell located in the opposite direction of the interface normal.

In a VoF/LS coupling, it is necessary to solve both of their transport equations simultaneously, the Level Set transport equation is defined as:

$$
\frac{\partial \phi}{\partial t}+\nabla \cdot\left(\phi \boldsymbol{u}_{l}\right)=0
$$

where $\phi$ is the signed distance function between a grid point and the interface. It takes positive values for the points in the liquid phase and negative for those in the gas. The discretization of both equations is made following the "coupled" second-order conservative operator split advection scheme defined in Sussman and Puckett (2000). The evaporation term in the rhs of eq. 26 is applied in the last step of the temporal integration of the liquid volume fraction.

The signed distance function is used to obtain the geometrical information of the interface, e.g., the vector normal to the interface $(\boldsymbol{n})$ and the curvature $(\kappa)$ :

$$
\boldsymbol{n}=\frac{\boldsymbol{\nabla} \phi}{|\boldsymbol{\nabla} \phi|} \quad \kappa=-\boldsymbol{\nabla} \cdot \boldsymbol{n}
$$

A redistancing algorithm is applied every iteration step following the same principle of previous work (Ménard et al. (2007), Tanguy et al. (2007), Duret 
et al. (2012)).

\subsection{Flow solver}

A projection method is used for the temporal resolution of the momentum equation. An Eulerian staggered grid is considered: the scalars variables $(P, T$, $\alpha_{l}$, etc) are defined on the cell center and velocities are located on the faces of the cell.

In this work, we are going to used the method proposed by (Tanguy et al. 2007) for a discontinuous velocity field at the interface. The Ghost Fluid Method (GFM) is used to populate the ghost cells on each side of the interface. The velocity extensions allow to have continuous liquid/gas velocity fields at the interface. As such, the numerical methods for the convective and diffusive terms of the Navier-Stokes equation, already implemented in the ARCHER code, can be used.

For the compressible part of this work, the projection method of (Tanguy et al. 2007) has been modified to take into account the compressible effects, i. e., density gradients due to an increase of pressure in an enclosed environment containing an evaporating liquid and spatial variation of the temperature field.

First, an intermediate velocity $\boldsymbol{u}^{*}$ is calculated for both liquid and gas field $\left(\boldsymbol{u}_{l}^{*}, \boldsymbol{u}_{g}^{*}\right)$ by solving the momentum equation (eq $1 \mathrm{~b}$ ) without the pressure:

$$
\left\{\begin{array}{l}
\boldsymbol{u}_{l}^{*}=\boldsymbol{u}_{l}^{n}-\Delta t\left(\left(\boldsymbol{u}_{l}^{n} \cdot \nabla\right) \boldsymbol{u}_{l}^{n}-\nabla \cdot\left(2 \mu \bar{\epsilon}_{l}-\frac{2}{3} \mu \boldsymbol{\nabla} \cdot \boldsymbol{u}_{l}^{n} \overline{\bar{I}}\right)\right) \\
\boldsymbol{u}_{g}^{*}=\boldsymbol{u}_{g}^{n}-\Delta t\left(\left(\boldsymbol{u}_{g}^{n} \cdot \nabla\right) \boldsymbol{u}_{g}^{n}-\nabla \cdot\left(2 \mu \overline{\bar{\epsilon}}_{g}-\frac{2}{3} \mu \boldsymbol{\nabla} \cdot \boldsymbol{u}_{g}^{n} \overline{\bar{I}}\right)\right)
\end{array}\right.
$$

the term $\frac{2}{3} \mu \boldsymbol{\nabla} \cdot \boldsymbol{u}^{n} \overline{\bar{I}}$ had been added to adapt the projection method to a compressible formalism. This new term is computed with a second order centered difference scheme. For equations 31 , the convective terms are computed with a 5th order WENO scheme and the viscous terms are solved with the method proposed by Sussman et al. (2007). Then, similar to the incompressible solver, the following strategy to update the velocity is applied:

$$
\boldsymbol{u}^{n+1}=\boldsymbol{u}^{*}-\Delta t \frac{\nabla P^{n+1}}{\rho^{n+1}}
$$


Then the divergence operator is applied:

$$
\boldsymbol{\nabla} \cdot \boldsymbol{u}^{n+1}=f-\boldsymbol{\nabla} \cdot\left(\Delta t \frac{\boldsymbol{\nabla} P^{n+1}}{\rho^{n+1}}\right)
$$

where

$$
f= \begin{cases}\boldsymbol{\nabla} \cdot \boldsymbol{u}_{l}^{*} & \text { if } \phi>0, \\ \boldsymbol{\nabla} \cdot \boldsymbol{u}_{g}^{*} & \text { if } \phi<0,\end{cases}
$$

The next step is to inject eq. 33 in the pressure equation (eq. 1c), yielding the following system for the pressure:

$$
\begin{aligned}
-\boldsymbol{\nabla} \cdot\left(\frac{\nabla P^{n+1}}{\rho^{n+1}}\right)+\left(\frac{1}{\rho c^{2} \Delta t^{2}}\right) P^{n+1} & =\left(\frac{1}{\rho c^{2} \Delta t^{2}}\right)\left(P^{n}+\Delta t \boldsymbol{u} \cdot \boldsymbol{\nabla} P^{n}\right) \\
& -\frac{f}{\Delta t}+\frac{\alpha_{T}}{\rho c_{p} \Delta t} \boldsymbol{\nabla} \cdot(\lambda \boldsymbol{\nabla} T)
\end{aligned}
$$

The convective term of the pressure $(\boldsymbol{u} \cdot \boldsymbol{\nabla} P)$ is discretized with a 5th order WENO scheme. The Poisson solver used for this Helmholtz type equation consists of an MGCG (MultiGrid preconditioned Conjugate Gradient) method coupled with the Gauss-Seidel "Red-Black" iterative scheme. The energy term of eq. 34 is computed during the evolution of the temperature; which is explained in the next section. Then, the real part of the velocity field of each phase is obtained with:

$$
\begin{cases}\boldsymbol{u}_{l}^{n+1}=\boldsymbol{u}_{l}^{*}-\Delta t \frac{\boldsymbol{\nabla} P^{n+1}}{\rho^{n+1}} & \text { if } \phi>0 \\ \boldsymbol{u}_{g}^{n+1}=\boldsymbol{u}_{g}^{*}-\Delta t \frac{\boldsymbol{\nabla} P^{n+1}}{\rho^{n+1}} & \text { if } \phi<0\end{cases}
$$

Now, in order to use the GFM, a liquid and gas velocity extensions must be estimated. For the liquid, a ghost pressure $\left(P^{\text {ghost }}\right)$ is computed:

$$
-\boldsymbol{\nabla} \cdot\left(\frac{\boldsymbol{\nabla} P^{g h o s t}}{\rho^{n+1}}\right)+\underbrace{\left(\frac{1}{\rho c^{2} \Delta t^{2}}\right) P^{\text {ghost }}}_{I}=-\frac{\boldsymbol{\nabla} \cdot \boldsymbol{u}_{l}^{*}}{\Delta t}+\underbrace{\frac{\alpha_{T}}{\rho c_{p} \Delta t} \boldsymbol{\nabla} \cdot(\lambda \boldsymbol{\nabla} T)}_{I I}
$$

Eq. 36 is solved following the same methods used for eq. 34. The new term I is related to the flow acoustics and the new term II is the contribution 
of the thermal dilatation. These two terms have been added compared to the original projection method proposed by Tanguy et al. (2007). The liquid velocity extension $\left(\boldsymbol{u}_{l}^{\text {ghost }}\right)$ is computed from $P^{\text {ghost }}$ :

$$
\boldsymbol{u}_{l}^{g h o s t}=\boldsymbol{u}_{l}^{*}-\Delta t \frac{\nabla P^{g h o s t}}{\rho^{n+1}}
$$

The above method is similar to (Tanguy et al., 2007). Here, both phases are considered to be compressible; for this reason, the steps dedicated to enforce the divergence-free condition in the liquid velocity field and its extension are skipped. It is worth mention that the liquid velocity extension $\left(\boldsymbol{u}_{l}^{\text {ghost }}\right)$ is continuous but non divergence-free. And the final liquid velocity is obtained with:

$$
\boldsymbol{u}_{l}^{n+1}= \begin{cases}\boldsymbol{u}_{l}^{n+1} & \text { if } \phi>0, \\ \boldsymbol{u}_{l}^{\text {ghost }} & \text { if } \phi<0,\end{cases}
$$

The velocity extension for the gas is computed by adding the interface velocity jump to the liquid velocity:

$$
\boldsymbol{u}_{g}^{g h o s t}=\boldsymbol{u}_{l}^{n+1}-s_{\boldsymbol{d}}\left(\frac{\rho_{l}-\rho_{g}}{\rho_{g}}\right)
$$

Finally, the gas velocity field is obtained:

$$
\boldsymbol{u}_{g}^{n+1}= \begin{cases}\boldsymbol{u}_{g}^{\text {ghost }} & \text { if } \phi>0, \\ \boldsymbol{u}_{g}^{n+1} & \text { if } \phi<0,\end{cases}
$$

With this formalism, two continuous velocity fields have been obtained in the whole domain, including at the interface location. Then, standard numerical methods for the convective transport for the momentum and scalars can be used directly.

\subsection{Energy transport}

For the evolution of the temperature field, in the energy equation (eq. 6), the term responsible for the energy generation by friction $(\overline{\bar{\tau}}: \nabla \boldsymbol{u})$ and the term 
of volumetric energy generation $(\dot{Q})$ are neglected because the production or consumption of energy by a chemical reaction in the system it is not considered, yielding:

$$
\rho c_{p}\left(\frac{\partial T}{\partial t}+\boldsymbol{u} \cdot \nabla T\right)=\nabla \cdot(\lambda \nabla T)+\alpha_{T} T\left(\frac{\partial P}{\partial t}+\boldsymbol{u} \cdot \nabla P\right)
$$

Eq. 41 is solved in two step. First, an intermediary temperature $\left(T^{*}\right)$ is computed containing the convective and diffusive terms. Then, the pressure term is added after the resolution of the Poisson equation.

The convective term of eq. 41 is discretized using a 5 th order WENO scheme. Strong temperature gradients can occur at the interface, which are a source of error and can lead to numerical heating. To avoid this, the temperature field of each phase is extrapolated using the Aslam's extension method (Aslam, 2004). The idea is to extend linearly the scalar of one phase, following the normal direction of the interface, into the other; populating the ghost grid points. Then a 5 th order WENO scheme is used for convection of the liquid temperature and its ghost values $\left(\boldsymbol{u}_{l} \cdot \nabla T_{l}\right)$ and the same procedure is applied for the gas side $\left(\boldsymbol{u}_{g} \cdot \boldsymbol{\nabla} T_{g}\right)$. Finally, these terms are introduced into eq. 41 depending on the sign of the Level Set function.

A 2nd order central difference scheme is used for the discretization of the diffusion term. When mass transfer is considered, the energy jump at the interface (eq. 18 must be taken into account in eq. 41. For this reason, the discretization scheme for the diffusion term is modified in the grid points close to the interface using a GFM.

\subsection{Vapor mass fraction transport}

For the transport of the vapor mass fraction $\left(Y_{v}\right)$, eq. 7 is solved only in the gas phase. $Y_{v}$ is also extended in the liquid phase with the Aslam's extension method. Eq. 7 reduces to:

$$
\frac{\partial Y_{v}}{\partial t}+(\boldsymbol{u} \cdot \boldsymbol{\nabla}) Y_{v}=\frac{\boldsymbol{\nabla} \cdot\left(\rho_{g} D_{m} \nabla Y_{v}\right)}{\rho_{g}}
$$


As for eq. 41, the diffusion term is discretized using a 2 nd order central difference scheme and the convective term is computed with a 5th order WENO scheme. To apply the Dirichlet boundary condition at the interface $\left(Y_{v s}\right)$, a 1st order Aslam-Chiu extension method is implemented. More details about this method are available in Bouali et al. (2016). The main idea of this method is to introduce a mirror point in gaseous phase, which is the image of the ghost point of $Y_{v}$ in the liquid, initially estimated with Aslam's extension method. Then, the mirror point is used for the correction of the estimation of the Aslam extension, following the value of the boundary condition given by eq. 21. The estimation of the ghost point of the vapor fraction $\left(Y_{v}^{G h}\right)$ in the liquid is computed as:

$$
Y_{v}^{G h}=2 Y_{v s}-\left(Y_{v}^{e x t}-2 \phi \frac{d Y_{v}}{d n}\right)
$$

where $Y_{v}^{e x t}$ is the first estimation of the ghost point, $\frac{d Y_{v}}{d n}$ is extracted from the linear Aslam's extension done before the convection step of $Y_{v}$ and $Y_{v s}$ is obtained with eq. 21 .

The interface temperature $\left(T^{\Gamma}\right)$ needed for eq. 20 is estimated with (in 1D):

$$
T^{\Gamma}=\frac{T_{l, i}\left|\phi_{i+1}\right|+T_{l, i+1}^{g h o s t}\left|\phi_{i}\right|}{\left|\phi_{i+1}\right|+\left|\phi_{i}\right|}
$$

where $T_{l}^{\text {ghost }}$ is the extended liquid temperature on the gas phase. Eq. 44 can be generalized into two and three dimensions. If the liquid and the gas temperatures are used instead of the liquid temperature and its extension to interpolate the interface temperature; unrealistic values can be obtained. Which will results in a erroneous determination of the Dirichlet boundary condition for $Y_{v}$ and the evaporation rate.

For both scalar fields ( $T$ and $Y_{v}$ ), when a grid node pass from being in one phase to the other due to the evolution of the interface, a correction step is implemented. During this step, $Y_{v}$ and $T$ of a 'switching' point $i$ are replaced with its ghost values given by the Aslam's extension method during the previous time step. The purpose of the correction is to avoid the artificial heating/cooling in regions close to the interface after the time integration of the interface capturing method. 
Fig. 1 show the algorithm used in a Euler time step for the compressible flow solver. The incompressible algorithm is not presented since it is similar to the one of Tanguy et al. (2007), but with a different interface capturing method (VOF). The VOF transport equation presented in Eq. 26 has been used to take into account evaporation processes in both formalism.

\section{Results (incompressible formalism)}

\subsection{Validation}

In this section two academic cases are presented to validate our incompressible formalism. First, a 2D static cylinder with an imposed evaporation rate is studied to demonstrate the accuracy of our sink term in the VoF equation, combined with the CLSVOF method for capturing reacting interfaces. Then, a $D^{2}$ law configuration is investigated to validate the implementation of the thermodynamics of the formalism as well as the coupling between the temperature, the vapor mass fraction and the evaporation rate. The physical properties for the air, water and n-decane are listed in table 1. The mass diffusion coefficient for the vapour in air is $D_{m}=2.1 \times 10^{-5} \mathrm{~m}^{2} \cdot \mathrm{s}^{-1}$ and $D_{m}=1 \times 10^{-5} \mathrm{~m}^{2} \cdot \mathrm{s}^{-1}$ for the n-decane vapour in air. The surface tension for water/air interface is $\sigma=0.07 N . m^{-1}$ and $\sigma=0.0135 N . m^{-1}$ for the n-decane/air interface.

\begin{tabular}{|c|ccccccc|}
\hline Fluid & $\rho\left(\frac{\mathrm{kg}}{\mathrm{m}^{3}}\right)$ & $\mu\left(\frac{\mathrm{kg}}{\mathrm{ms}}\right)$ & $\lambda\left(\frac{\mathrm{W}}{\mathrm{mK}}\right)$ & $C_{p}\left(\frac{\mathrm{J}}{\mathrm{kgK}}\right)$ & $M\left(\frac{\mathrm{kg}}{\mathrm{mol}}\right)$ & $h_{l v}\left(\frac{\mathrm{J}}{\mathrm{kg}}\right)$ & $T_{B}(K)$ \\
\hline Air & 1.226 & $1.78 \times 10^{-5}$ & 0.046 & 1000 & 0.029 & & \\
Water & 1000 & $1.137 \times 10^{-3}$ & 0.6 & 4180 & 0.018 & $2.3 \times 10^{6}$ & 373 \\
N-decane & 750 & $5.65 \times 10^{-4}$ & 0.14 & 2207 & 0.142 & $3.25 \times 10^{5}$ & 447 \\
\hline
\end{tabular}

Table 1: Physical properties for the air, water and n-decane

\subsubsection{Interface regression}

A 2D static water cylinder with a constant evaporation rate is used to validate the estimation of the sink term in the VoF equation (eq. 26) responsible 


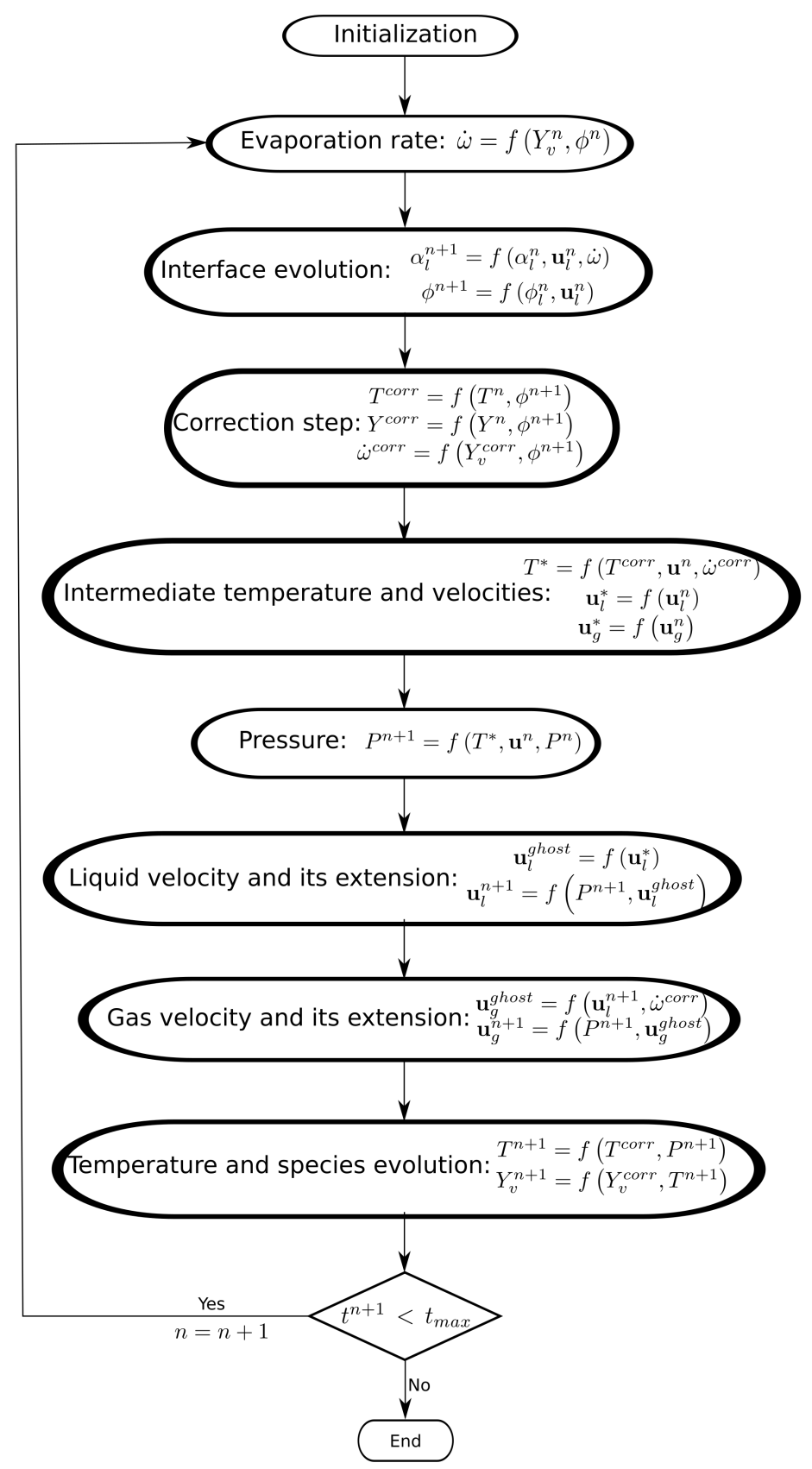

Figure 1: Summary of a euler time step for the compressible flow solver. 
for the regression of the interface due to phase change and the efficiency of the aforementioned liquid velocity extension method. Since the evaporation rate is imposed, the energy and species conservation equations are not considered in this configuration. However, the Navier-Stokes equations are solved.

The initial cylinder radius is $R_{D}=150 \mu \mathrm{m}$ and the domain is a square with a side length of $l_{x}=l_{y}=8 R_{D}$. Initially, the velocity of both phase is equal to zero and outflow boundary conditions are employed in all directions. Theoretically, the evolution of the cylinder mass $\left(m_{t h}\right)$ is given by:

$$
m_{t h}(t)=\rho_{l} \pi\left(R_{D}-\left\|\boldsymbol{s}_{\boldsymbol{d}}\right\| t\right)^{2}
$$

The interface regression speed $\left\|\boldsymbol{s}_{\boldsymbol{d}}\right\|$ is set to $10^{-3} \mathrm{~m} \cdot \mathrm{s}^{-1}$. Simulations are made for four mesh sizes: $32^{2}, 48^{2}, 64^{2}$ and $128^{2}$.

One of the difficulties with this configuration is the magnitude of the spurious currents in the liquid velocity field. Spurious currents are created during computation of $\boldsymbol{u}_{l}$ and depend of the numerical method used for the treatment of the interface velocity jump. If the magnitude of the spurious velocities is important, unrealistic deformation of the interface will occur; leading to issues in the mass conservation. For the static configuration the liquid velocity and its extension are supposed to be zero $\left(\boldsymbol{u}_{l}=\boldsymbol{u}_{l}^{\text {ghost }}=0\right)$, the regression velocity $\left(\boldsymbol{s}_{\boldsymbol{d}}\right)$ is the only one influencing the interface evolution. On the other hand, the real part of the gas velocity $\left(\boldsymbol{u}_{g}\right)$ should contain the Stefan flow around the interface. The different velocities are shown in fig 2 : a) the liquid velocity field with its extension in the gas phase; b) the gas velocity field and its extension in the liquid phase; c) the real velocity field. Here, we can see that the magnitude of the spurious currents in the liquid velocity field are several order of magnitude smaller than the velocity of the gas. Consequently, good agreement with the theoretical evolution of the cylinder mass and the simulation results is obtained.

The simulations are stopped after $36 \%$ of the initial cylinder mass have been evaporated. In Fig. 3 the normalized cylinder mass evolution $\left(m^{*}=\frac{m_{l}}{m_{l}^{0}}\right)$ for a constant evaporation rate is shown. In Fig. 3 a we can see that there is a good agreement between the simulations and the theoretical results and in Fig. 3b 


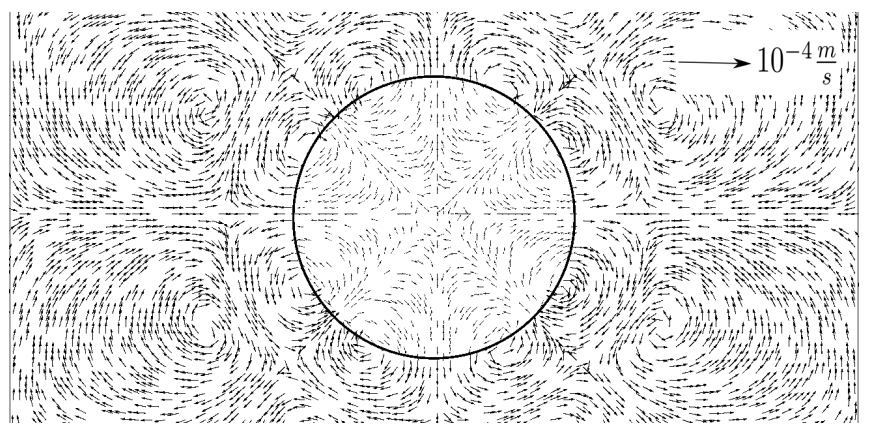

(a)

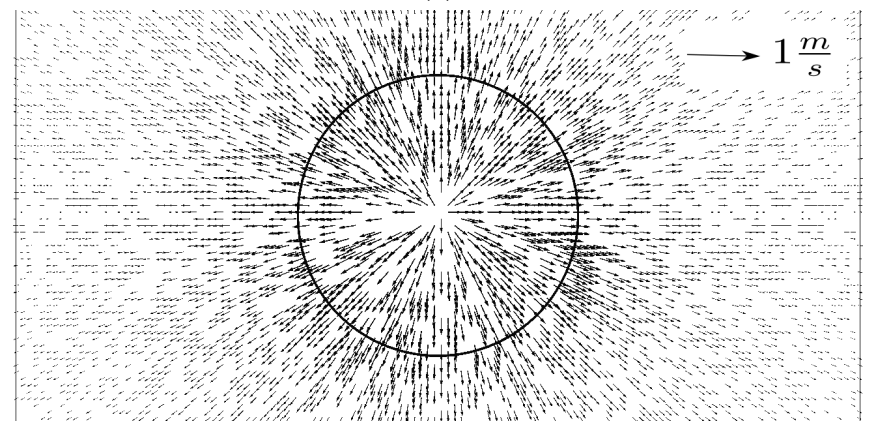

(b)

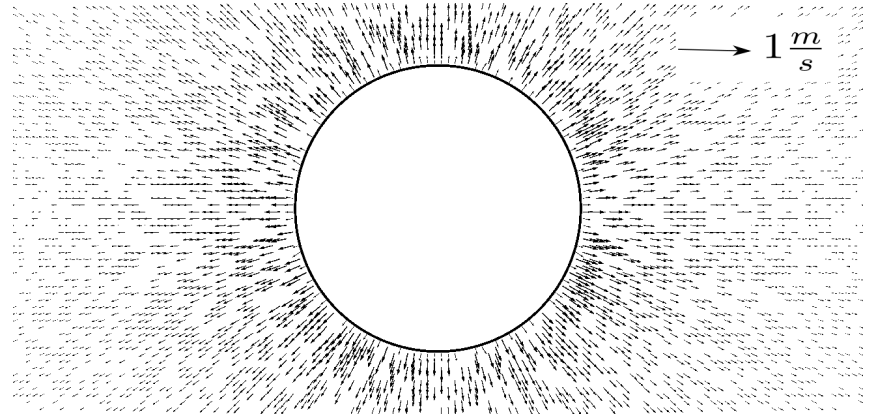

(c)

Figure 2: Velocity fields and its extensions $(t=0.001 \mathrm{~s})$. a): Liquid, b): gas, c) real. 
the simulations converge to the theoretical results as the grid mesh is refined. To go further in the grid convergence analysis, the $L_{p}$ norms are plotted in Fig. 4. The $L_{p}$ norms are computed as:

$$
\begin{aligned}
L_{\infty} & =\max \left|m_{t h}(t)-m(t)\right| \\
L_{1} & =\frac{\sum\left|m_{t h}(t)-m(t)\right|}{N} \\
L_{2} & =\sqrt{\frac{\sum\left|m_{t h}(t)-m(t)\right|^{2}}{N}}
\end{aligned}
$$

A order of convergence close to 2 has been obtained. These results show an improvement in both the magnitude of the relative errors and in the order of convergence compared with the results obtained by Tanguy et al. (2007). This difference can be explained by the use of a conservative method for the interface capturing (CLSVOF method).

\subsection{2. $D^{2}$ law}

In order to validate the implementation of the thermodynamics in the incompressible formalism, a static droplet configuration is compared with the well-known $D^{2}$ law. According to this law, the square of the droplet diameter decreases linearly with time because of heat and mass diffusion in the surrounding gas film (Sirignano and Edwards, 2000). To obtain the normalized square radius equation, the continuity equation in spherical coordinates must be solved assuming incompressibility and a constant liquid mass flux from the interface to the surrounding air $\left(\dot{m}^{\prime}=4 \pi r^{2} \rho \boldsymbol{u}\right)$.

Among the assumptions necessary for the $D^{2}$ law to be applicable, the isolated droplet must be in an infinite continuous medium. Using this condition into a numerical domain is too computationally expensive. Because, it would imply that the interface of the droplet is sufficiently far from the the domain boundary, in order to not disturb the spatial profile of the velocity, vapor mass fraction or temperature. To be representative of the finite domain simulation, we integrate the species and energy conservation equations using the values of 


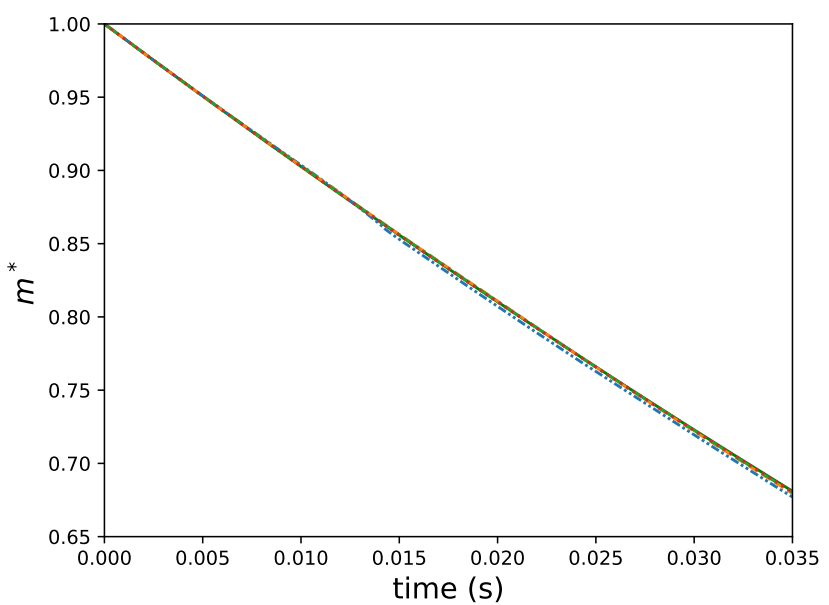

(a)

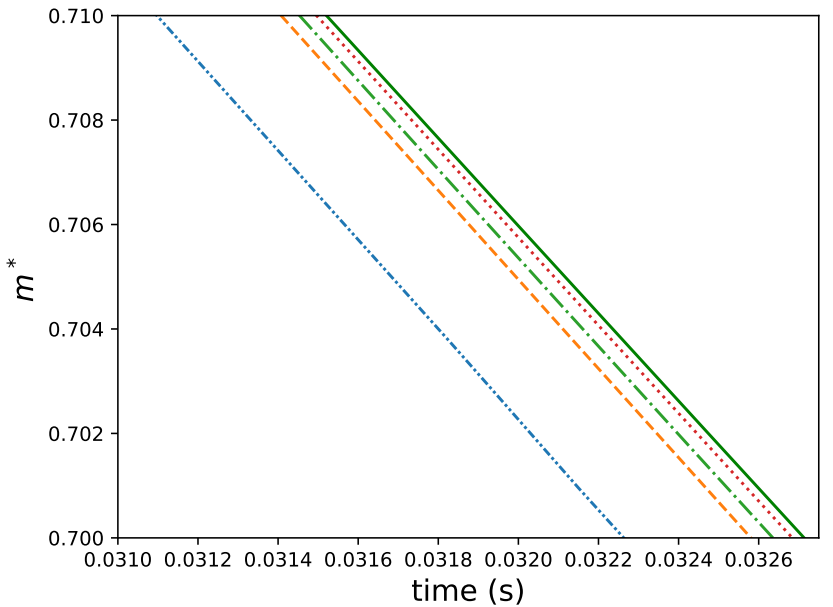

(b)

Figure 3: Liquid mass evolution for a constant evaporation rate. Solid: Theoretical solution, Dashdotdotted: $32^{2}$, Dashed: $48^{2}$, Dashdot: $64^{2}$, Dotted: $128^{2}$. 


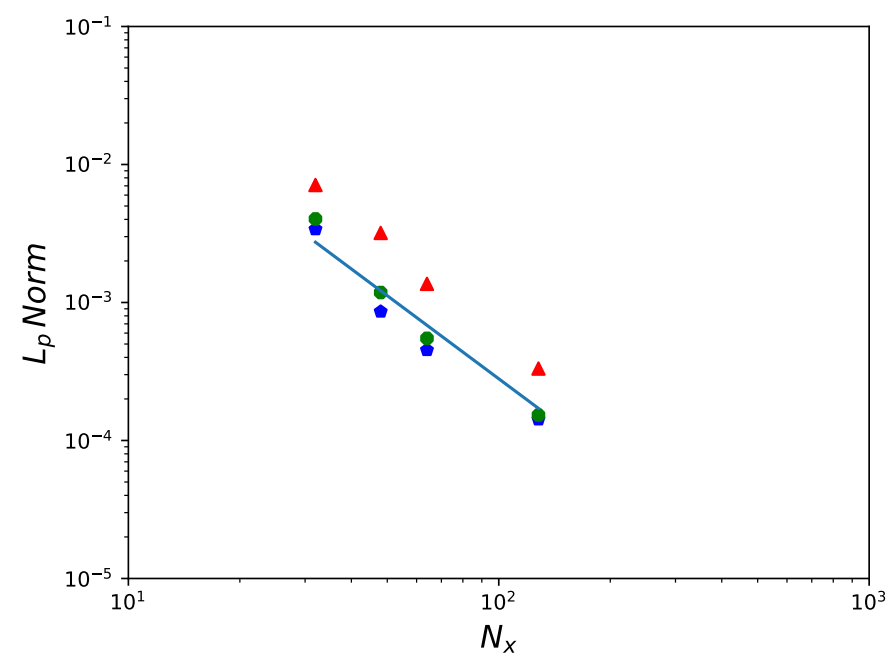

Figure 4: $L_{\infty}, L_{1}$ and $L_{2}$ norm of the liquid mass temporal evolution for a constant evaporation rate. Solid line: 2nd order, $\triangle: L_{\infty}, \circ: L_{1}, \bullet: L_{2}$.

each variable at the limit of the numerical domain, yielding:

$$
\begin{gathered}
Y_{v}(r)=1+\left(Y_{v B C}-1\right) \exp \left[-\frac{\dot{m}^{\prime}}{4 \pi \rho D_{m}}\left(\frac{1}{r}-\frac{1}{r_{B C}}\right)\right] \\
\ln \left(\frac{T(r)-\frac{h_{l v}}{c_{p}}-\frac{\varphi}{\dot{m}^{\prime}}}{T_{B C}-\frac{h_{l v}}{c_{p}}-\frac{\varphi}{\dot{m}^{\prime}}}\right)=-\frac{\dot{m}^{\prime}}{4 \pi \rho D_{g}^{t}}\left(\frac{1}{r}-\frac{1}{r_{B C}}\right)
\end{gathered}
$$

where $Y_{v B C}, T_{B C}$ are the vapor mass fraction and temperature at the boundary of the domain, respectively. $r_{B C}$ is the distance from the center of the droplet to the limit of the domain. $D_{m}$ is the mass diffusivity. $D_{g}^{t}=\frac{\lambda}{\rho_{g} c_{p g}}$ is the thermal diffusivity. $\dot{m}^{\prime}$ is computed by evaluating eq. 49 at the interface $\left(Y_{v}\left(R_{D}\right)=Y_{v s}\right)$ :

$$
\dot{m}^{\prime}=-4 \pi \rho \frac{r r_{B C}}{r_{B C}-r} \ln \left(\frac{Y_{v s}-1}{Y_{v \infty}-1}\right)
$$

And $\varphi$ can be interpreted as the heat flow entering the liquid. $\varphi$ is obtained by evaluating eq. 50 at the interface $\left(T\left(R_{D}\right)=T_{\Gamma}\right)$ :

$$
\varphi=-\frac{\dot{m}\left(h_{l v}+\exp \left[-\frac{\dot{m}}{4 \pi \rho D_{g}^{t}}\left(\frac{1}{r}-\frac{1}{r_{B C}}\right)\right]\left(T_{B C} c_{p}-h_{l v}\right)-T_{\Gamma}\right)}{c_{p}\left(1-\exp \left[-\frac{\dot{m}}{4 \pi \rho D_{g}^{t}}\left(\frac{1}{r}-\frac{1}{r_{B C}}\right)\right]\right)}
$$


This equation considers the length of the numerical domain in our theoretical calculation, making the results more comparable.

This configuration is particularly difficult because we are trying to reproduce the theoretical results given by solving the conservation equations in spherical coordinates using a Cartesian mesh. To compare the vapor mass fraction and temperature profiles computed by eq. 49 and 50 with the simulation results, it is necessary to wait enough time for the configuration to go from the initial conditions to a stationary state. In other words, the vapor mass fraction and temperature temporal derivative must be small enough to be considered as negligible. To lower the computational cost and prevent the movement of the droplet from the center of the domain, $\boldsymbol{u}_{l}=0 . s_{\boldsymbol{d}}$ is the only velocity responsible for the interface evolution.

A water droplet with initial radius of $R_{D}=150 \mu \mathrm{m}$ is considered. The initial droplet temperature is $T_{l}=353 K$ and the initial gas temperatures is $T_{g}=573 K$. The domain is a cube with a side length of $8 R_{D}$. Simulations are carried out with three mesh sizes: $32^{3}, 48^{3}$ and $64^{3}$. Outflow boundary conditions are employed in all directions.

On Fig. 5. the temperature (a) and the vapor mass fraction (b) are presented at $t=0.08 \mathrm{~s}$ for the finest grid $\left(64^{3}\right)$. As expected, a spherical symmetric property around the droplet is observed for both fields. The temporal evolution of the normalized diameter of the droplet is showed in Fig. 8 In the first moments of the simulation, a strong regression speed is observed. This is because the liquid is not initialized at the equilibrium temperature. Then the curve start to stabilize, resulting in a linear trend as the temperature at the interface approach an equilibrium temperature; as established in the $D^{2}$ law. This results show the ability of our method to handle strong temperature gradients at the interface and the strong coupling between the vaporization rate and the fluid dynamics efficiently. Fig. 7 and Fig. 6 shows the temperature and vapor mass fraction profile in the gas phase obtained by solving eq. 50 and 49 and the profiles obtain with three simulations $\left(32^{3}, 48^{3}\right.$ and $\left.64^{3}\right)$ as a function of the 


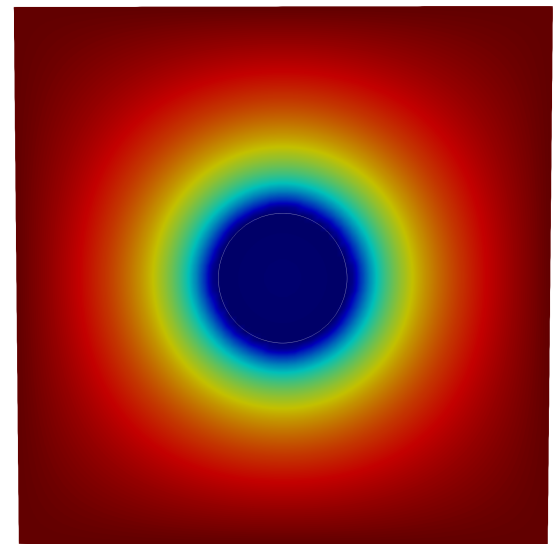

(a)

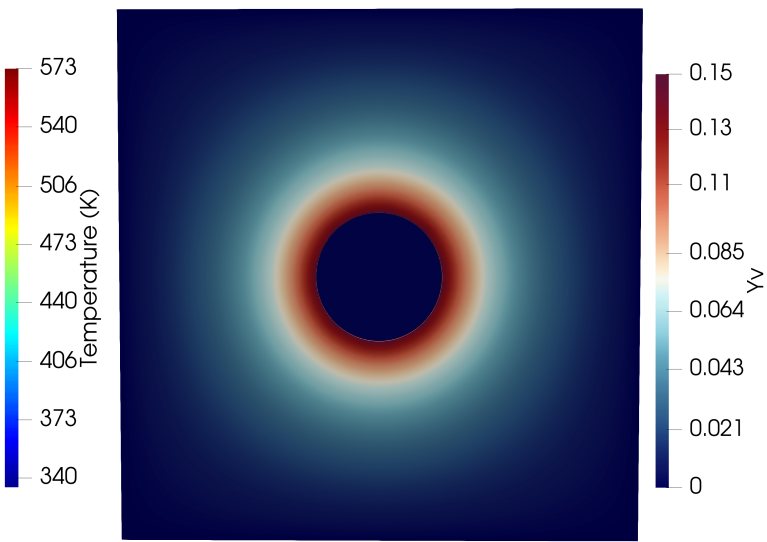

(b)

Figure 5: (a) Temperature field and (b) vapor mass fraction field $(t=0.08 s)$.

normalized radius $\left(r^{*}=\frac{r}{R_{D}}\right)$. A good agreement with the theory is found for all the simulations.

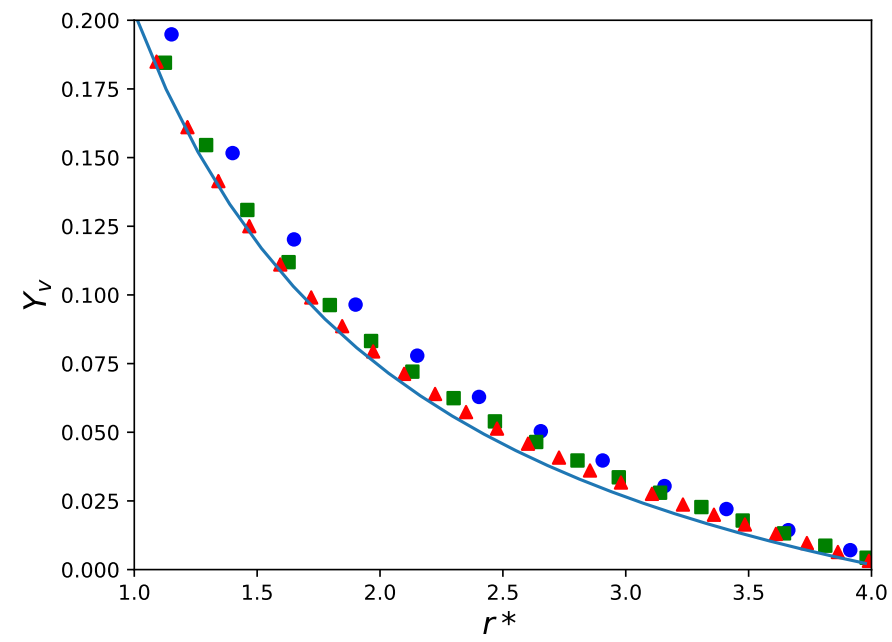

Figure 6: Radial vapor mass fraction profile in the gaseous phase $(t=0.08 \mathrm{~s})$. Solid line: theoretical solution of $D^{2}$ law, $\circ: 32^{3}, \square: 48^{3} \triangle: 64^{3}$. 


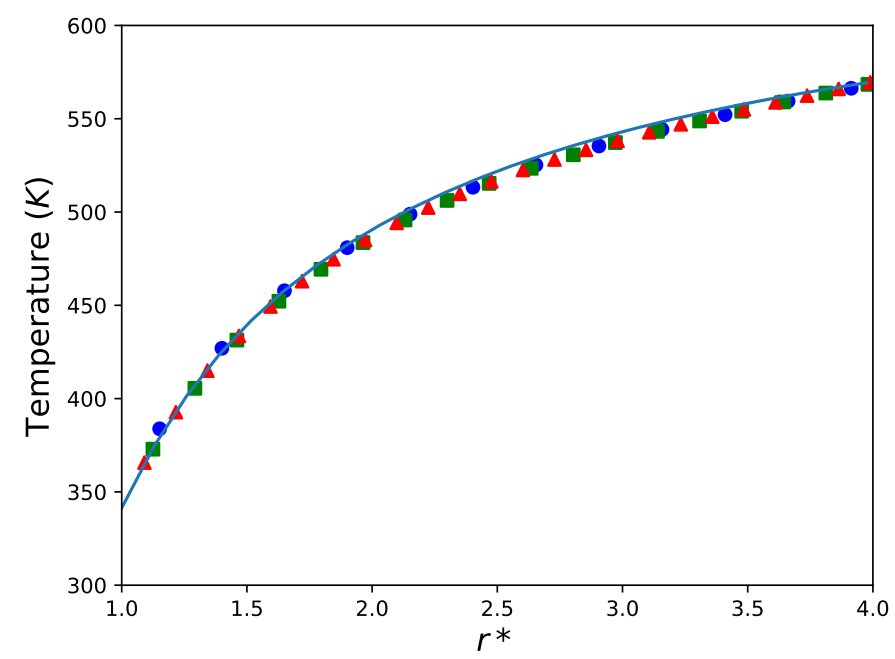

Figure 7: Radial temperature profile in the gaseous phase $(t=0.08 \mathrm{~s})$. Solid line: theoretical solution of $D^{2}$ law, o: $32^{3}, \square: 48^{3} \triangle: 64^{3}$.

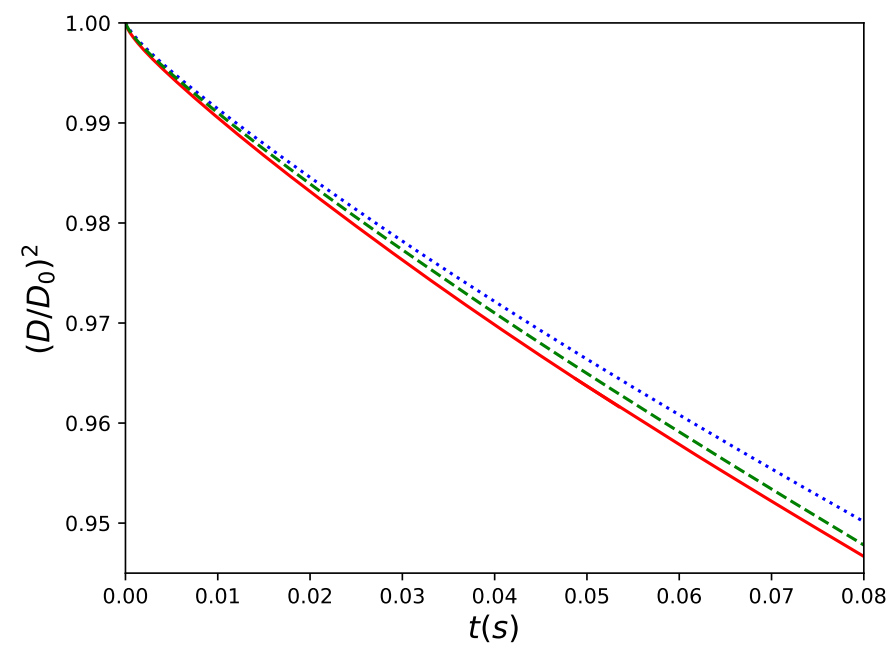

Figure 8: Normalized square diameter versus time $(\mathrm{s})$ for a $T_{g}=573 \mathrm{~K}$. Solid: $64^{3}$, Dashed: $48^{3}$, Dotted: $32^{3}$. 


\subsection{Convected evaporating cylinder}

In this section, a simulation of an evaporating falling water cylinder is presented. This configuration illustrates the formalism ability to handle the convection term in the Navier-Stokes solver in the presence of discontinuities in the velocity field combined with the CLSVOF interface capturing method. The initial cylinder radius is $R_{D}=300 \mu \mathrm{m}$ and the initial liquid velocity, gas velocity, liquid and gas temperature are $\boldsymbol{u}_{l}=1 \mathrm{~m} . \mathrm{s}^{-1}, \boldsymbol{u}_{g}=0 \mathrm{~m} . \mathrm{s}^{-1}, T_{l}=323 \mathrm{~K}$ and $T_{g}=873 \mathrm{~K}$, respectively. Outflow boundary conditions are used in all directions. For the temperature and vapor mass fraction, Dirichlet boundary conditions are imposed: $T=873 K$ and $Y v=0$. A 2D rectangular domain is considered with a length in the x-direction $l_{x}=8 R_{D}$ and in the y-direction $l_{y}=4 l_{x}$. Simulations are carried out with a $128 \times 512$ grid size.

Fig. 9 shows the temperature and the vapor mass fraction field at $t=$ $6 \mathrm{~ms}$. The numerical methods implemented in this work for the treatment of the different jump conditions and strong temperature gradients at the interface, allow us to capture several phenomena. For example, we can identify the thermal and species boundary layers formed around the cylinder. In the front, the presence of stronger temperature and vapor mass fraction gradients than in the back of the cylinder shows the formation of a recirculation zone due to the moderate cylinder Reynolds number investigated $\left(R e_{D}=\frac{\rho_{g} D u_{i n i t}}{\mu_{\text {gas }}}=40\right)$. This recirculation zone causes a homogenization of the temperature and vapor mass fraction behind the cylinder, forming a plume in both fields. The variation of the vapor mass fraction around the cylinder and the temperature at the interface induces a non-homogeneous evaporation rate (See Fig. 10). This result highlights the importance of considering the local interface temperature and the local surface density for computing of the evaporation rate.

Fig. 11 shows the streamlines, colored with the temperature, of the gas flow behind the cylinder and the iso-contour of $\boldsymbol{u}_{g}=0$ for two simulations: on the right, the evaporating falling cylinder; on the left, we have the same configuration, but this time, without evaporation (heating only). An axisymmetric vortex and a slight detachment and enlargement of the recirculation zone is observed 


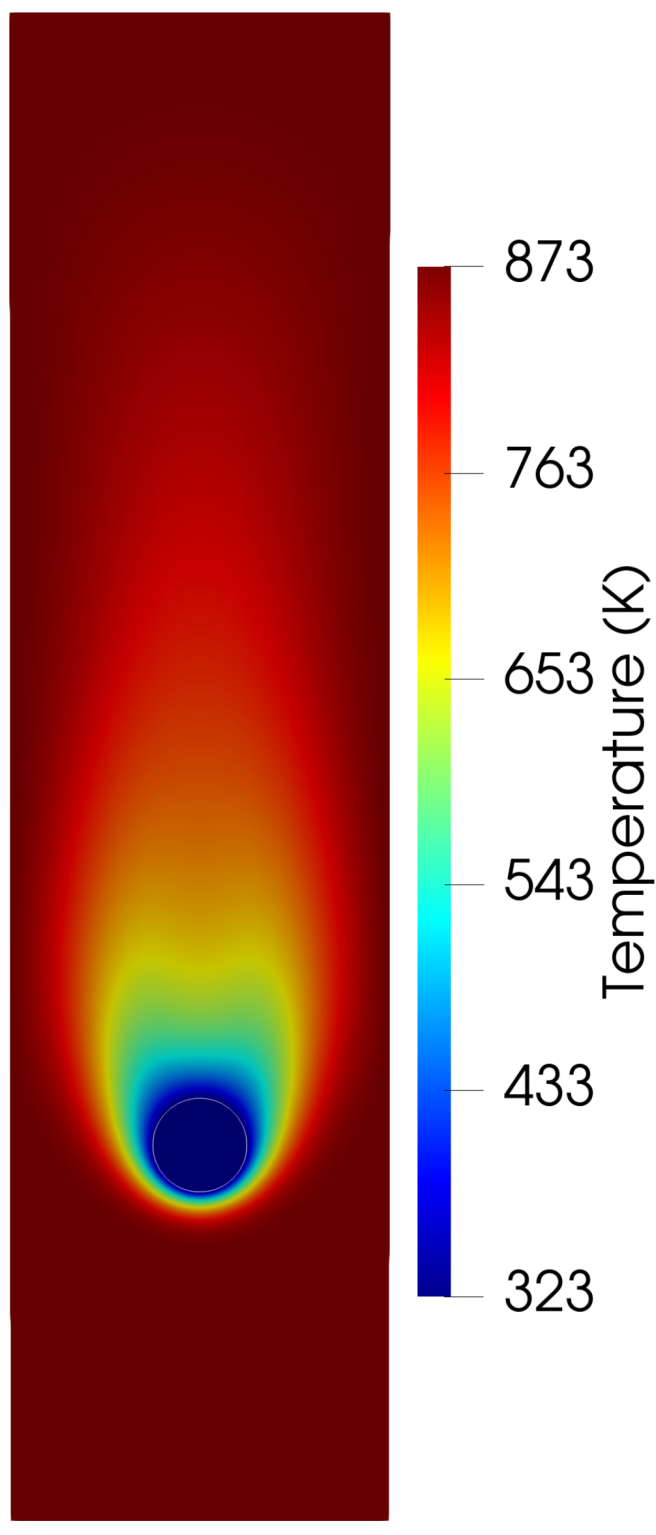

(a)

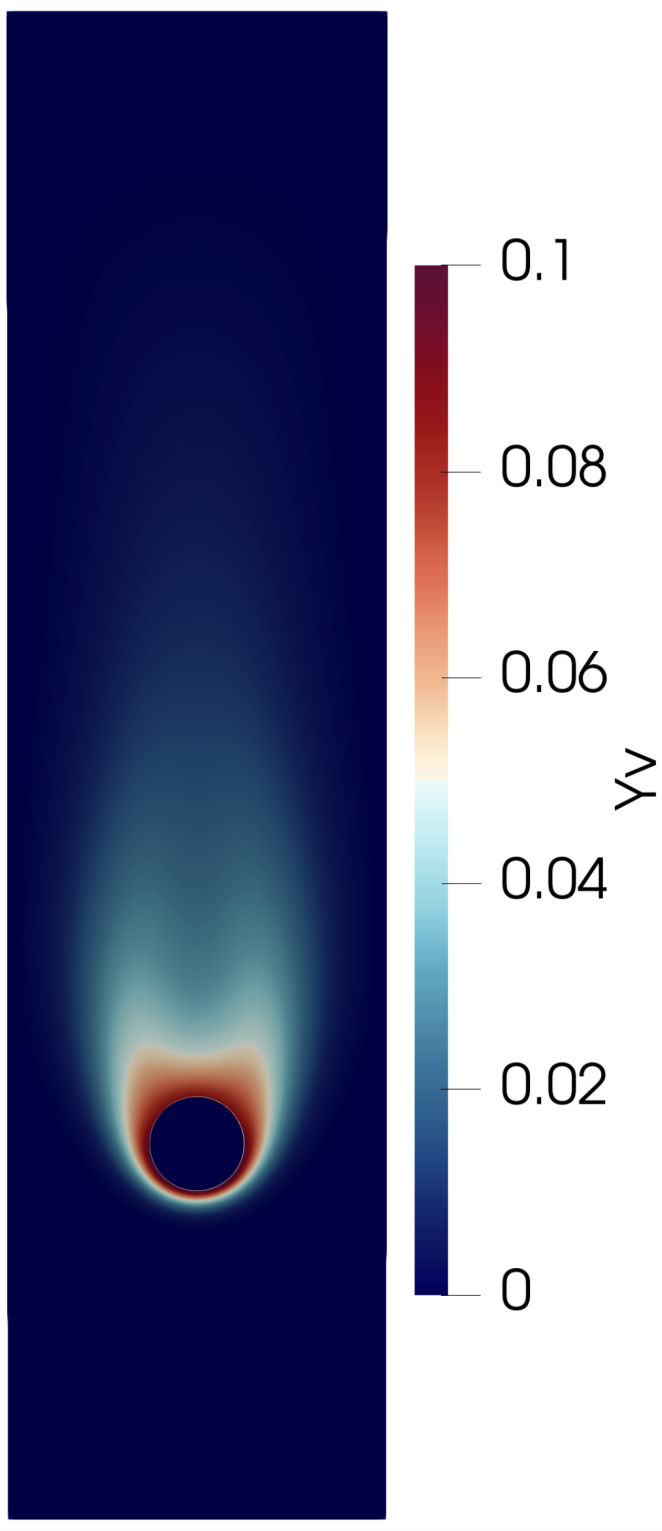

(b)

Figure 9: (a) Temperature field and (b) vapor mass fraction field $(t=6 \mathrm{~ms})$ for a initial velocity of $\boldsymbol{u}_{l}=1 \frac{\mathrm{m}}{\mathrm{s}}$. 


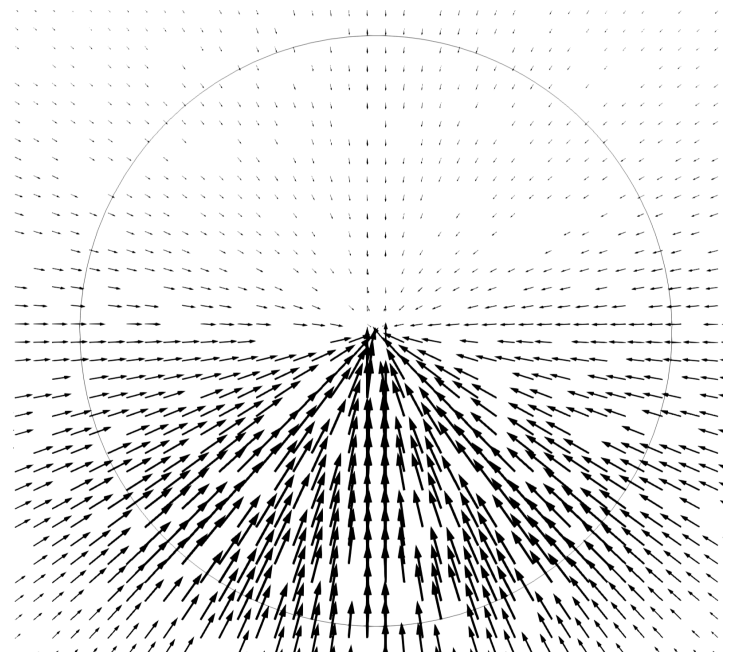

Figure 10: Normal evaporation rate $(\dot{\omega} \boldsymbol{n})$ and its extension around the drop $(t=6 \mathrm{~ms})$. $\rightarrow: 0.025 \frac{\mathrm{kg}}{\mathrm{m}^{2} \mathrm{~s}}$.

in the back of the evaporating cylinder. This is explained by the presence of the Stefan flow; which creates an envelope around the interface and separates the airflow from the cylinder.

To validate the fluid dynamics behavior, the normalized length of the recirculation zones $\left(L^{*}=\frac{L}{D_{c y l}}\right)$ for both evaporating and non-evaporating simulations has been compared with those found in Dennis and Chang (1970) for a solid cylinder. A good agreement is found for the non-evaporating water cylinder with $1.4 \%$ of difference with the reference, while the evaporating configuration have a difference of $4.7 \%$.

To go further in the demonstration of the influence of the Stefan flow in the characteristic of the recirculation zone, two additional simulations are performed for a n-decane cylinder, while keeping the same Reynolds number. Fig. 12 shows the streamlines of the flow behind of the n-decane cylinder: on the right, the evaporating configuration and on the left, the non-evaporating one. Here, we can see that the detachment of the recirculation zone is more pronounced. Due to its thermophysical properties, the Stefan flow is stronger for the ndecane for the same temperature difference between the initial temperature of 

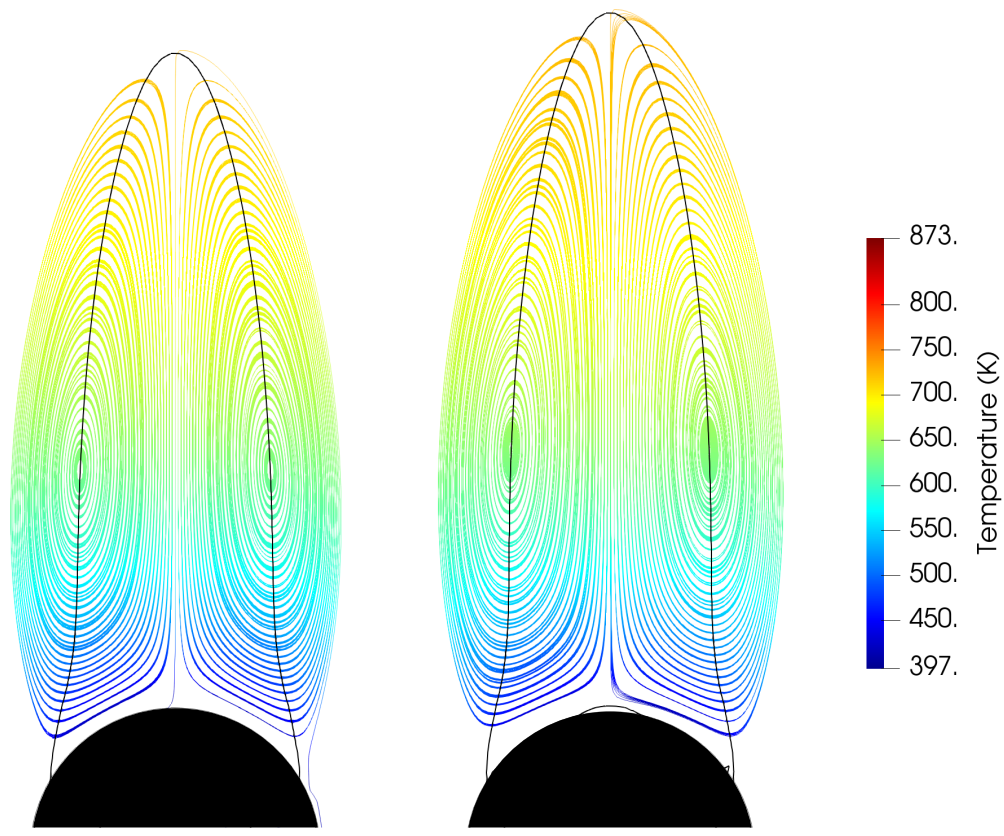

Figure 11: Velocity streamlines behind the water cylinder and visualisation of the $\boldsymbol{u}=0$ iso-contour. Right: evaporating drop, left: non-evaporating drop.

the liquid and its boiling temperature $\left(\Delta T=T_{B}-T_{l i q}\right)$. This time, a difference with the reference value of almost $32 \%$ is found for the evaporating n-decane cylinder. Results are summarized in table 2 It is worth mention that the same phenomenon of detachment of the recirculation zone from the back of the interface and its enlargement was also observed by Jayawickrama et al. (2019) and Jayawickrama et al. (2021). They studied the effect of the Stefan flow on the drag coefficient and the Nusselt number of a spherical particle in a constant airflow. 


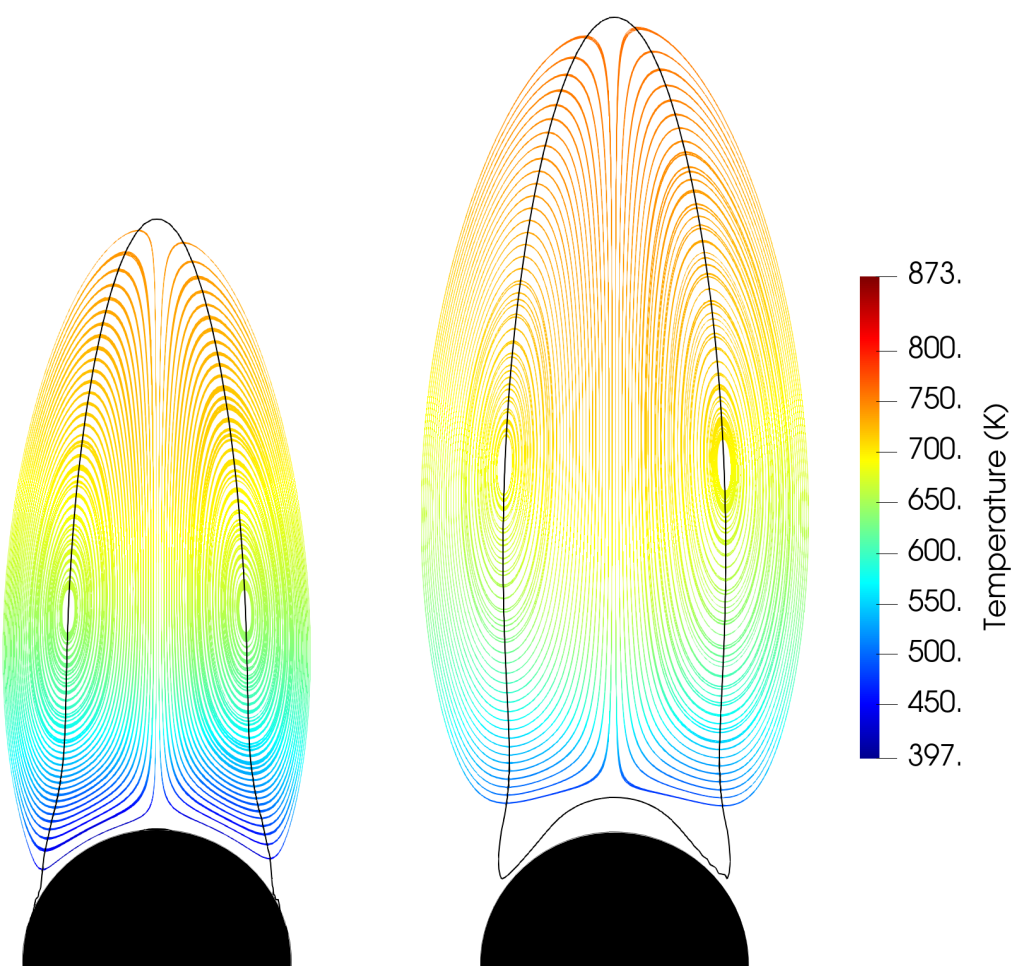

Figure 12: Velocity streamlines behind the n-decane cylinder and visualisation of the $\boldsymbol{u}=0$ iso-contour. Right: evaporating drop, left: non-evaporating drop.

\begin{tabular}{|c|ccccc|}
\hline Liquid & Evaporation & $R e$ & $L^{*}$ & Ref. & $\%$ Diff. \\
\hline Water & off & 40 & 4.62 & 4.69 & 1.4 \\
Water & on & 40 & 4.91 & 4.69 & 4.7 \\
N-decane & off & 40 & 4.65 & 4.69 & 0.85 \\
N-decane & on & 40 & 6.20 & 4.69 & 32 \\
\hline
\end{tabular}

Table 2: Comparison of the length of the recirculation zone for two different liquid. 


\section{Results (compressible formalism)}

The following results use the compressible formalism presented in Section 3.

\subsection{Mass conservation in enclosed environment}

In this section, the same configuration of section 4.2 is used (static water cylinder configuration), but this time the objective is to validate the compression and dilatation effects due to evaporation in two-phase flows. To simulate a enclosed environment, periodic boundary conditions are employed in all directions. This configuration cannot be performed with an incompressible solver: in this kind of formalism, the Stefan flow generated at the interface (dilatation) should be evacuated by at least one outflow boundary condition in one direction. In a Low Mach or a weakly-compressible framework, the divergence created will increase the pressure and the gas density.

The initial cylinder radius is $R_{D}=200 \mu \mathrm{m}$. A $2 \mathrm{D}$ rectangular domain is considered with a side length of $l_{x}=24 R_{D}$. Simulations are performed with a $512^{2}$ grid resolution. In this configuration, the vaporization is imposed at $\dot{\omega}=1 \frac{\mathrm{kg}}{\mathrm{m}^{2} \mathrm{~s}}$. The temperature and the species transport equation are not resolved in this subsection.

With a prescribed vaporization rate, the temporal derivative of the total gas mass is $\frac{\partial m_{g}}{\partial t}=\dot{\omega} S$. Consequently, an estimation of the total mass gas at a given instant $n+1\left(m_{g}^{n+1}\right)$, including the dilatation induced by the Stefan flow, can be written as :

$$
m_{g}^{n+1}=m_{g}^{n}+\dot{\omega} S^{n} \Delta t
$$

where $m_{g}^{n}$ is the total gas mass at the previous timestep of the simulation. Note that the result given by eq. 53 are not theoretical because the local interface area $S_{P L I C}$ from the PLIC reconstruction is extracted in each cell and then summed to obtain the total surface $S$. Fig. 13 shows the evolution of the normalized gas mass $\left(m_{g}^{*}=\frac{m_{g}(t)}{m_{g}^{0}}\right)$ obtained in the simulation where $m_{g}^{0}$ is the initial total gas mass, and compared with the reference results given by eq. 53. A good agreement is found between the simulation results and the reference; which 
validate the coupling between the velocity jump term in the Poisson pressure equation and the phase change sink term in the Vof equation. For completeness, Fig. 14 shows the simulation results for the evolution of the liquid mass and the reference results computed in a similar way with:

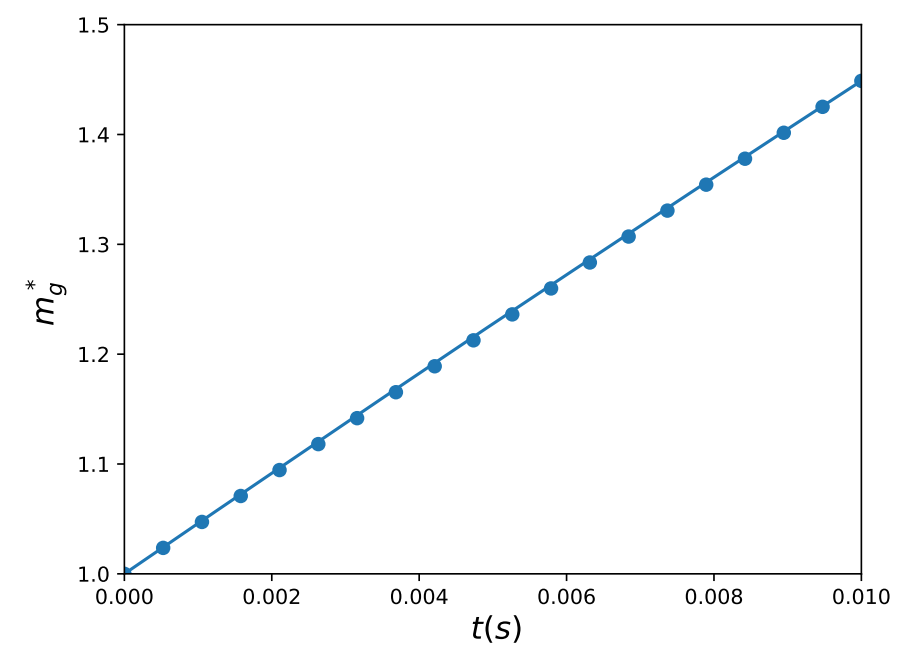

Figure 13: Gas mass temporal evolution. Solid line: Reference solution, o: simulation results.

$$
m_{l}^{n+1}=m_{l}^{n}-\dot{\omega} S^{n} \Delta t
$$

Furthermore, the total mass in a enclosed environment should remain constant during all the simulation to respect continuity. In this configuration, the total mass shows a reduction of less than $0.02 \%$ at the end of the simulation for the finest mesh resolution (See Table 3). This result demonstrates the excellent mass conservation properties of our CLSVOF interface capturing method.

\begin{tabular}{|c|c|c|c|c|}
\hline Mesh & $96^{2}$ & $192^{2}$ & $384^{2}$ & $512^{2}$ \\
\hline E (\%) & 0.058 & 0.024 & 0.035 & 0.017 \\
\hline
\end{tabular}

Table 3: Errors for the non-dimensional total mass for three mesh sizes in $t=0.01 \mathrm{~s}$ 


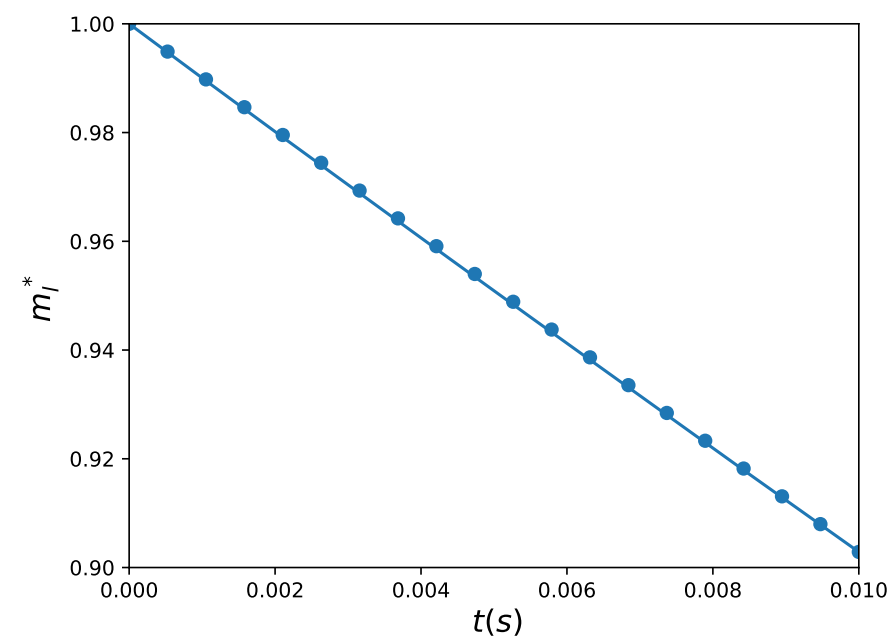

Figure 14: Liquid mass temporal evolution. Solid line: Reference solution, o: simulation results.

\subsection{Compressible HIT configuration with phase change}

Similar to previous works, the idea is to study the influence of the evaporation phenomenon inside a forced two-phase flows HIT. In Duret et al. (2012), a passive scalar was used to represent the evaporation and mixing process. Among the limitations of this procedure, the influence of the Stefan flow in the velocity field and the increase of the pressure due to the evaporation process were not considered. A major improvement has been proposed by Duret et al. (2018) where a phase change source term was directly introduced in the continuity and pressure equations. The results obtained illustrated good mass conservation properties. However, the influence of the temperature in the vapor mass fraction at the interface was not implemented yet to the general formalism: the vaporization rate was directly imposed. Contrary to Duret et al. (2018), here, the velocity jump is introduced implicitly to the Poisson equation for the pressure in the ghost points of the gas velocity field. The coupling between the energy, the continuity and momentum equations is added by assuming thermodynamic equilibrium at the interface.

The thermodynamic properties are those of n-decane (table 1). The initial 
gas density is $\rho_{g}=25 \mathrm{~kg} \cdot \mathrm{m}^{-3}$. The pressure is $P=4.11 \times 10^{6} \mathrm{~Pa}$ and for the Tait equation, the parameters used for the equation of state are $B=10^{9} \mathrm{~Pa}$, $P_{0}=10^{5} \mathrm{~Pa}, \rho_{0}=750 \mathrm{~kg} \cdot \mathrm{m}^{-3}$ and $\gamma_{l}=1.215$. The initial liquid volume fraction is $\Phi=10 \%$ and the targeted mean kinetic energy is $\bar{k}=3.6 \frac{\mathrm{m}^{2}}{\mathrm{~s}^{2}}$. The initial liquid temperature is $T_{l}=340 \mathrm{~K}$ and the initial gas temperatures is $T_{g}=573 \mathrm{~K}$. The domain is a cube with a side length of $1.5 \times 10^{-4} \mathrm{~m}$ and a $256^{3}$ mesh has been retained for this simulation.

Fig. 15 shows a instantaneous temperature field and the iso-contour of the level-set function which represent the liquid/gas interface. As well as in other compressible and incompressible HIT configurations presented in previous works, many breakup and coalescence events are observed. Also, small gas structures are observed inside the liquid; these structures are characterized by a increase in pressure due to the creation of vapor and a decrease in temperature because of the energy consumption induced by the phase change. Additionally, the influence of the convection due to the turbulent velocity field in the temperature field is observed. Similar phenomena are found in the vapor mass fraction field (Fig. 16).

To observe the compressibility and dilatation effects on the velocity field, the velocity divergence is shown on Fig. 17 (top) in the gas phase. By comparing Fig. 17 (top) and Fig. 15, an increase of the velocity divergence has been observed in the presence of temperature gradients in the gas phase. This phenomenon can be explained by rewriting Eq. 1c to obtain the equation of the velocity divergence:

$$
\boldsymbol{\nabla} \cdot \boldsymbol{u}=-\frac{1}{\rho c^{2}} \frac{D P}{D t}+\frac{\alpha_{T}}{\rho c_{p}}(\boldsymbol{\nabla} \cdot(\lambda \nabla T))
$$

where the second term is the thermal dilatation. This term is dominant in the velocity divergence budget in the largest gas structure, meaning that the acoustic/compressible effects (first term) have a lower order of magnitude in this structure. However, the divergence inside encapsulated gas structure is generated differently: compressible effects have a major role in this scenario. The Stefan flow compresses the gas in a very small volume, leading to an increase 
of the divergence and of the gas density. This effect is illustrated in Fig. 17 (bottom): the divergence inside the encapsulated gas structure is almost one order of magnitude larger than the maximum divergence in the main gas structure. The temperature inside this structure is quasi uniform. Peaks of velocity divergence are also observed close to the interface, due to the presence of the Stefan flow (velocity jump).

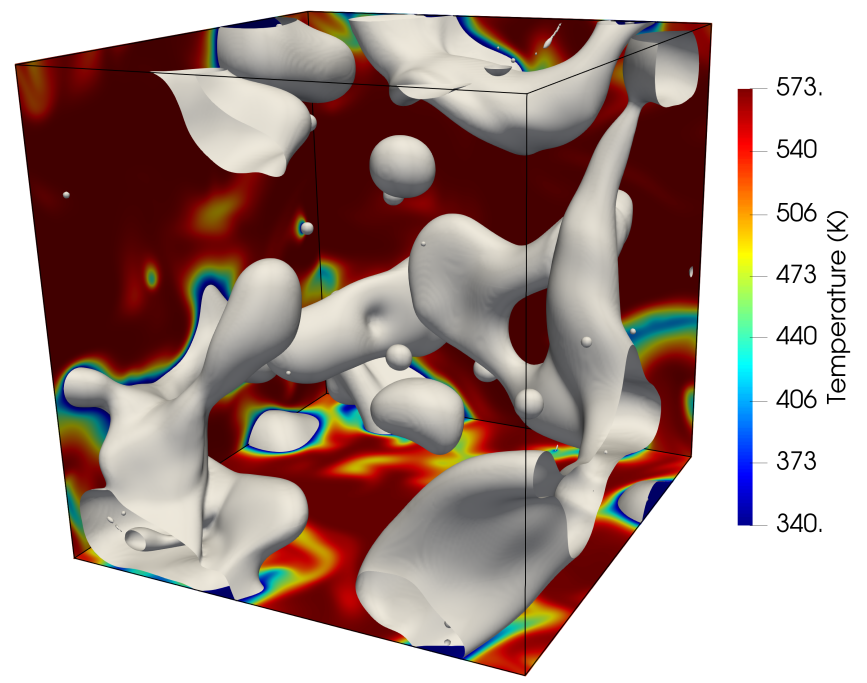

Figure 15: Instantaneous temperature field with interface location (Level Set 0 isocontour). 


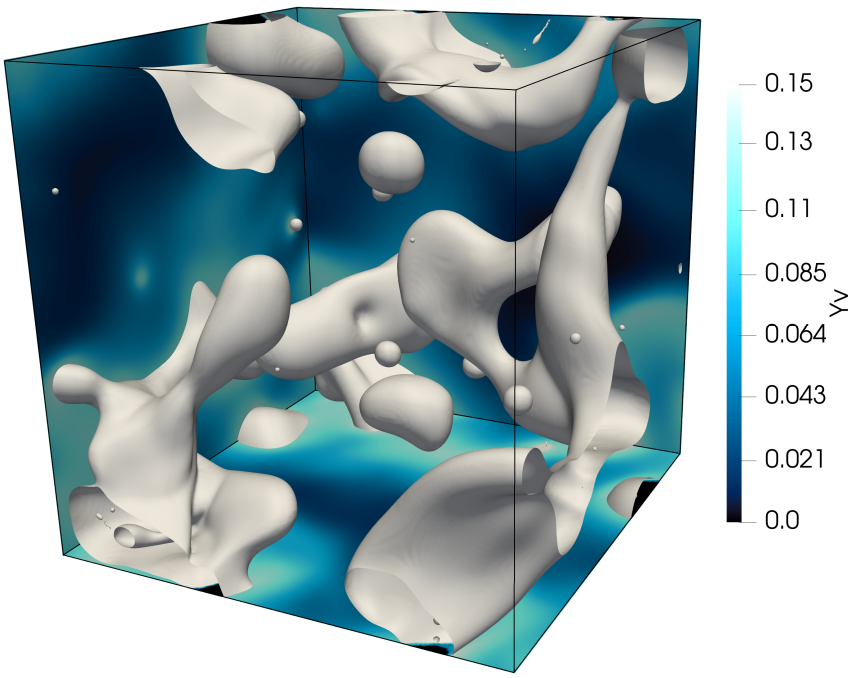

Figure 16: Instantaneous vapor mass fraction field with interface location (Level Set 0 isocontour). 

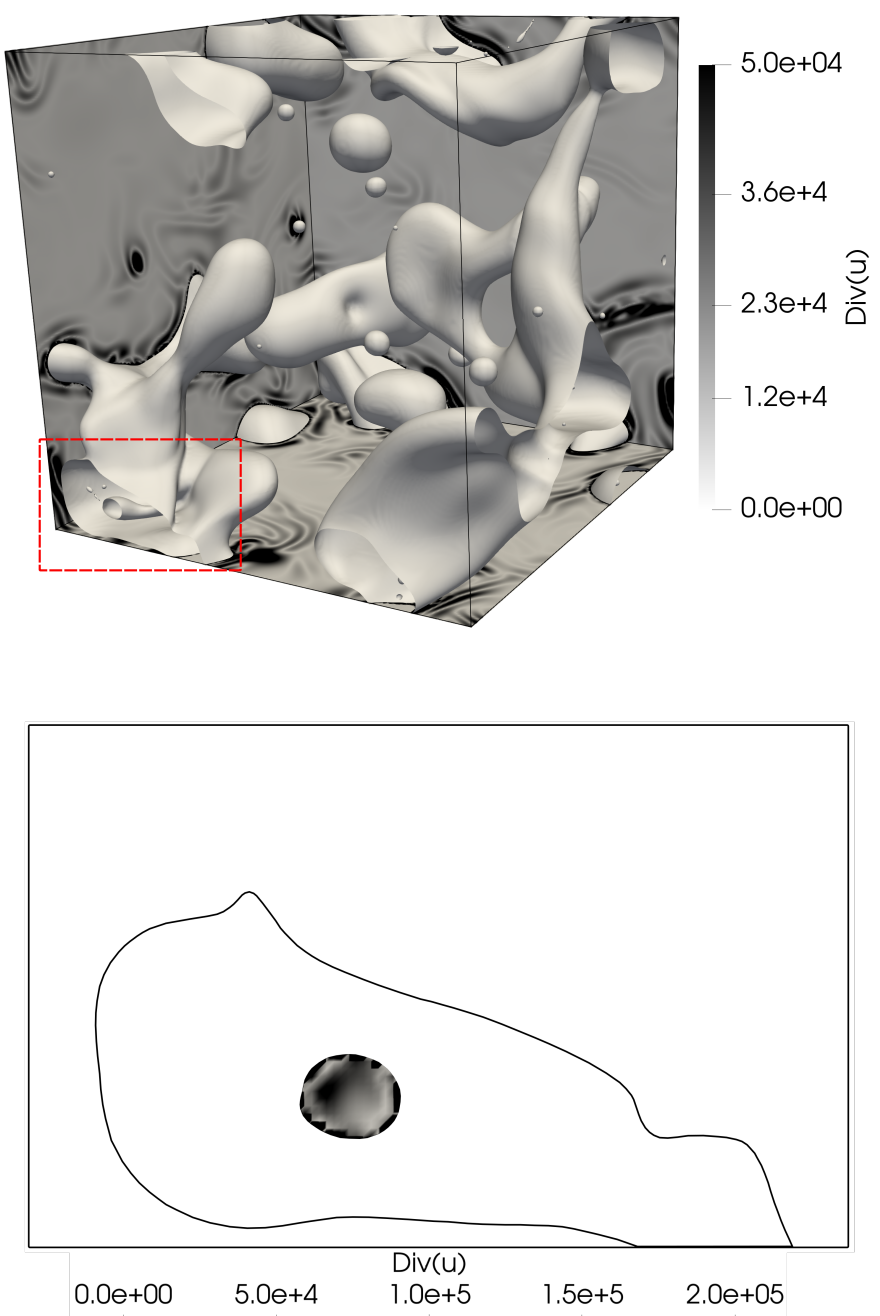

Figure 17: Top: instantaneous divergence of the real velocity field with interface location (Level Set 0 isocontour). Bottom: Visualization of the velocity divergence inside the encapsulated gas structure highlighted in red in the top figure. 


\section{Conclusion}

The development of a pressure based method for compressible multiphase flows with phase change is presented. First, an incompressible formalism for simulating of two-phase vaporizing flows is coupled with a mass conservative interface capturing method. A volume sink term in the VOF equation, based on the surface density, is used to represent the evaporation process. The use of accurate extensions for the discontinuous variables and the ghost fluid method allows the description of the additional jump conditions at the interface due to heat and mass transfer. These techniques are able to handle strong temperature gradients while avoiding numerical diffusion and artificial heating inside the liquid. Several validations cases are shown to illustrate the mass conservation properties of our method and the quality of the coupling between the vaporization rate and the flow dynamic. A discussion on numerical methods dedicated to two-phase flows with phase change is also presented to emphasize the main difficulties related to the implementation of phase change in a one fluid formalism and to address solutions to overcome it.

Then, the aforementioned numerical methods are extended to a weakly compressible formalism. Compressible Navier-Stokes equations and additional terms related to the compressiblity of the flow, such as thermal dilatation or the presence of a velocity divergence, are implemented. Additionally, the VOF equation is modified to consider the liquid compressibility, the variable density and the evaporation process. A validation case in an evaporating static cylinder configuration is investigated. A comparison between the results obtained with the compressible framework and with a reference equation representing the mass balance in an enclosed environment is performed, showing the accuracy of the formalism.

Finally, the latter formalism is used to simulate a 3D compressible HIT configuration and present the ability of our method to handle strong interface curvature, variable density, thermal dilatation, encapsulation of gas structure with their own thermodynamic pressure, collisions and breakups. Future work 
will be dedicated to the study of the interaction of the Stefan flow with the turbulence characteristics.

\section{Acknowledgements}

This work was granted access to the HPC resources of IDRIS, TGCC and CINES under the allocation A0092B10101 made by GENCI (Grand Equipement National de Calcul Intensif). The CRIANN (Centre Regional Informatique et d'Applications Numeriques de Normandie) is also gratefully acknowledged (project No. 2017004) for their CPU resources. Also, the authors thank the MESCYT (Ministerio de Educación Superior, Ciencia y Tecnología, Dom. Rep.) and professor Bruno Renou who have supported and managed this work in the framework of the Caliope program.

\section{References}

Akhmetbekov, Y.K., Alekseenko, S.V., Dulin, V.M., Markovich, D.M., Pervunin, K.S., 2010. Planar fluorescence for round bubble imaging and its application for the study of an axisymmetric two-phase jet. Experiments in Fluids 48, 615-629.

Aslam, T.D., 2004. A partial differential equation approach to multidimensional extrapolation. Journal of Computational Physics 193, 349-355.

Bilsky, A., Lozhkin, Y.A., Markovich, D., 2011. Interferometric technique for measurement of droplet diameter. Thermophysics and aeromechanics 18, 112.

Birouk, M., Gökalp, I., 2006. Current status of droplet evaporation in turbulent flows. Progress in energy and combustion science 32, 408-423.

Bouali, Z., Duret, B., Demoulin, F.X., Mura, A., 2016. Dns analysis of smallscale turbulence-scalar interactions in evaporating two-phase flows. International Journal of Multiphase Flow 85, 326-335. 
Calimez, X., 1998. Simulation a petite échelle par une méthode VOF d'écoulements diphasiques réactifs. Ph.D. thesis. Châtenay-Malabry, Ecole centrale de Paris.

Caltagirone, J.P., Vincent, S., Caruyer, C., 2011. A multiphase compressible model for the simulation of multiphase flows. Computers \& fluids 50, 24-34.

Chauveau, C., Birouk, M., Gökalp, I., 2011. An analysis of the d2-law departure during droplet evaporation in microgravity. International journal of multiphase flow $37,252-259$.

Chauveau, C., Birouk, M., Halter, F., Gökalp, I., 2019. An analysis of the droplet support fiber effect on the evaporation process. International Journal of Heat and Mass Transfer 128, 885-891.

Dennis, S., Chang, G.Z., 1970. Numerical solutions for steady flow past a circular cylinder at reynolds numbers up to 100. Journal of Fluid Mechanics $42,471-489$.

Dodd, M.S., Mohaddes, D., Ferrante, A., Ihme, M., 2021. Analysis of droplet evaporation in isotropic turbulence through droplet-resolved dns. International Journal of Heat and Mass Transfer 172, 121157.

Duret, B., Canu, R., Reveillon, J., Demoulin, F., 2018. A pressure based method for vaporizing compressible two-phase flows with interface capturing approach. International Journal of Multiphase Flow 108, 42-50.

Duret, B., Luret, G., Reveillon, J., Ménard, T., Berlemont, A., Demoulin, F.X., 2012. Dns analysis of turbulent mixing in two-phase flows. International Journal of Multiphase Flow 40, 93-105.

Gibou, F., Chen, L., Nguyen, D., Banerjee, S., 2007. A level set based sharp interface method for the multiphase incompressible navier-stokes equations with phase change. Journal of Computational Physics 222, 536-555. 
Guion, A., Afkhami, S., Zaleski, S., Buongiorno, J., 2018. Simulations of microlayer formation in nucleate boiling. International Journal of Heat and Mass Transfer 127, 1271-1284.

Hirt, C.W., Nichols, B.D., 1981. Volume of fluid (vof) method for the dynamics of free boundaries. Journal of computational physics 39, 201-225.

Huber, G., Tanguy, S., Béra, J.C., Gilles, B., 2015. A time splitting projection scheme for compressible two-phase flows. Application to the interaction of bubbles with ultrasound waves. Journal of Computational Physics 302, 439468.

Ishii, M., Hibiki, T., 2010. Thermo-fluid dynamics of two-phase flow. Springer Science \& Business Media.

Jayawickrama, T.R., Haugen, N.E.L., Babler, M.U., Chishty, M., Umeki, K., 2021. The effect of stefan flow on nusselt number and drag coefficient of spherical particles in non-isothermal gas flow. International Journal of Multiphase Flow , 103650 .

Jayawickrama, T.R., Haugen, N.E.L., Babler, M.U., Chishty, M.A., Umeki, K., 2019. The effect of stefan flow on the drag coefficient of spherical particles in a gas flow. International Journal of Multiphase Flow 117, 130-137.

Khalloufi, M., Valette, R., Hachem, E., 2020. Adaptive eulerian framework for boiling and evaporation. Journal of Computational Physics 401, 109030.

Kristyadi, T., Deprédurand, V., Castanet, G., Lemoine, F., Sazhin, S.S., Elwardany, A., Sazhina, E., Heikal, M., 2010. Monodisperse monocomponent fuel droplet heating and evaporation. Fuel 89, 3995-4001.

Kwatra, N., Su, J., Grétarsson, J.T., Fedkiw, R., 2009. A method for avoiding the acoustic time step restriction in compressible flow. Journal of Computational Physics 228, 4146-4161. 
Malan, L., Malan, A., Zaleski, S., Rousseau, P., 2020. A geometric vof method for interface resolved phase change and conservative thermal energy advection. arXiv preprint arXiv:2001.03477 .

Maqua, C., Castanet, G., Grisch, F., Lemoine, F., Kristyadi, T., Sazhin, S., 2008. Monodisperse droplet heating and evaporation: experimental study and modelling. International Journal of Heat and Mass Transfer 51, 39323945.

Mikami, M., Oyagi, H., Kojima, N., Kikuchi, M., Wakashima, Y., Yoda, S., 2005. Microgravity experiments on flame spread along fuel-droplet arrays using a new droplet-generation technique. Combustion and flame 141, 241252 .

Mirjalili, S., Jain, S.S., Dodd, M., 2017. Interface-capturing methods for twophase flows: An overview and recent developments. Center for Turbulence Research Annual Research Briefs 2017, 117-135.

Ménard, T., Tanguy, S., Berlemont, A., 2007. Coupling level set/VOF/ghost fluid methods: Validation and application to $3 \mathrm{~d}$ simulation of the primary break-up of a liquid jet. International Journal of Multiphase Flow 33, 510524.

Nguyen, D.Q., Fedkiw, R.P., Kang, M., 2001. A boundary condition capturing method for incompressible flame discontinuities. Journal of Computational Physics 172, 71-98.

Palmore Jr, J., Desjardins, O., 2019. A volume of fluid framework for interfaceresolved simulations of vaporizing liquid-gas flows. Journal of Computational Physics 399, 108954.

Popescu, E.R., Tanguy, S., Colin, C., 2019. On the influence of liquid/vapor phase change onto the nusselt number of a laminar superheated or subcooled vapor flow. International Journal of Thermal Sciences 140, 397-412. 
Reutzsch, J., Kieffer-Roth, C., Weigand, B., 2020. A consistent method for direct numerical simulation of droplet evaporation. Journal of Computational Physics 413, 109455.

Scapin, N., Barba, F.D., Lupo, G., Rosti, M.E., Duwig, C., Brandt, L., 2021. Finite-size evaporating droplets in weakly-compressible homogeneous shear turbulence. arXiv preprint arXiv:2104.10184 .

Scapin, N., Costa, P., Brandt, L., 2020. A volume-of-fluid method for interfaceresolved simulations of phase-changing two-fluid flows. Journal of Computational Physics 407, 109251.

Schlottke, J., Weigand, B., 2008. Direct numerical simulation of evaporating droplets. Journal of Computational Physics 227, 5215-5237.

Sirignano, W.A., Edwards, C.F., 2000. Fluid dynamics and transport of droplets and sprays. J. Fluids Eng. 122, 189-190.

Strizhak, P., Volkov, R., Castanet, G., Lemoine, F., Rybdylova, O., Sazhin, S., 2018. Heating and evaporation of suspended water droplets: Experimental studies and modelling. International Journal of Heat and Mass Transfer 127, $92-106$.

Sussman, M., Puckett, E.G., 2000. A coupled level set and volume-of-fluid method for computing 3d and axisymmetric incompressible two-phase flows. Journal of computational physics 162, 301-337.

Sussman, M., Smereka, P., Osher, S., 1994. A level set approach for computing solutions to incompressible two-phase flow. Journal of Computational physics $114,146-159$

Sussman, M., Smith, K.M., Hussaini, M.Y., Ohta, M., Zhi-Wei, R., 2007. A sharp interface method for incompressible two-phase flows. Journal of Computational Physics 221, 469-505. 
Tanguy, S., Ménard, T., Berlemont, A., 2007. A level set method for vaporizing two-phase flows. Journal of Computational Physics 221, 837-853.

Tomar, G., Biswas, G., Sharma, A., Agrawal, A., 2005. Numerical simulation of bubble growth in film boiling using a coupled level-set and volume-of-fluid method. Physics of Fluids 17, 112103.

Urbano, A., Tanguy, S., Colin, C., 2019. Direct numerical simulation of nucleate boiling in zero gravity conditions. International Journal of Heat and Mass Transfer 143, 118521.

Verwey, C., Birouk, M., 2017. Experimental investigation of the effect of droplet size on the vaporization process in ambient turbulence. Combustion and Flame 182, 288-297.

Verwey, C., Birouk, M., 2018. Fuel vaporization: Effect of droplet size and turbulence at elevated temperature and pressure. Combustion and Flame 189, 33-45.

Wang, Y., Chen, X., Wang, X., Yang, V., 2019. Vaporization of liquid droplet with large deformation and high mass transfer rate, ii: Variable-density, variable-property case. Journal of Computational Physics 394, 1-17.

Wang, Y., Yang, V., 2019. Vaporization of liquid droplet with large deformation and high mass transfer rate, i: Constant-density, constant-property case. Journal of Computational Physics 392, 56-70.

Wu, Y., Promvongsa, J., Saengkaew, S., Wu, X., Chen, J., Gréhan, G., 2016. Phase rainbow refractometry for accurate droplet variation characterization. Optics letters 41, 4672-4675. 